\title{
Initial Stage of Fiber Structure Development in the Continuous Drawing of Poly(ethylene terephthalate)
}

Keywords: fiber structure development, poly(ethylene terephthalate), synchrotron x-ray scattering, time-resolution, smectic-like structure

\section{Authors: Takayoshi Yamaguchi, Takahisa Murata, Mitsuharu Koide, Shuuji Hitoosa, Hiroshi Urakawa, Yutaka Ohkoshi, Yasuo Gotoh, Masanobu Nagura, Masaru Kotera,}

\section{Kanji Kajiwara}

\begin{abstract}
The initial stage of fiber structure development in the continuous neck-drawing of amorphous poly(ethylene terephthalate) fibers was analyzed by in-situ wide-angle $\mathrm{x}$-ray diffraction, small-angle $x$-ray scattering, and temperature measurements. The time error of the measurements $(<600 \mu \mathrm{s})$ was obtained by synchrotron $\mathrm{x}$-ray source and laser irradiation heating. A highly ordered fibrillar-shaped two-dimensional (smectic-like) structure was found to be formed less than $1 \mathrm{~ms}$ after necking. By analyzing its (001') and (002') diffractions, the length of the structure 60-70 $\mathrm{nm}$ were obtained. A three-dimensionally ordered triclinic crystal began to form with the vanishing of the structure around $1 \mathrm{~ms}$ after necking. The amount and size of the crystal were almost saturated within several milliseconds of necking, during which time a mainly exothermic heat of crystallization was also observed.
\end{abstract}

\section{Introduction}

Poly(ethylene terephthalate) (PET; $\left.\left.\left[-\mathrm{O}-\mathrm{CO}-\mathrm{C}_{6} \mathrm{H}_{4}-\mathrm{CO}-\mathrm{O}-\left(\mathrm{CH}_{2}\right)_{2}\right)-\right]_{\mathrm{n}}\right)$ is a semi-crystalline polymer that has a triclinic crystal system ${ }^{1)}$. Although it is the most widely used polyester, its high-ordered structure and the relationship between its properties and structure remain unclear. While various structural models ${ }^{2)-6)}$ have 
been proposed for semi-crystalline polymers, the mechanical properties of a fiber cannot be estimated quantitatively by structural parameters. This is because the whole shape of a molecular chain cannot be expressed in terms of the structural parameters obtained for a produced fiber. The fiber structure, i.e., the characteristic structure in which the crystal and amorphous phases align along the fiber axis in alternating fashion, is formed during the melt spinning and/or drawing processes with the molecular chains' extension and instantaneous orientation-induced crystallization. Thus, if we can analyze the fiber structure development process, we can predict the shape of a molecular chain more accurately and estimate, for example, the amount and state of the load-bearing structure, and thus design the fiber properties more quantitatively.

As a result, there are many reports on in-situ x-ray measurement that analyze the fiber structure development process. However, it is difficult to analyze the initial stage of fiber structure development because the orientation-induced crystallization is completed within a few milliseconds in the typical manufacturing process of PET fibers. In the case of melt spinning, there are some reports on the high-speed spinning line, dealing with the x-ray analysis of fiber structure development ${ }^{17)-21)}$. In particular, the report by Haberkorn et al. ${ }^{18)}$, who took x-ray images of polyamide 66 fibers for a running speed of $5500 \mathrm{~m} / \mathrm{min}$, is important of its $0.16 \mathrm{~ms}$ time resolution, which can be estimated from the reported fluctuation width of the necking position of about $3 \mathrm{~cm}$. In addition, in the case of PET, Kolb et al. ${ }^{19)}$ reported x-ray images with a corresponding time precision of $\pm 0.3 \mathrm{~ms}$ for 4000 - $\mathrm{m} / \mathrm{min}$ high-speed spinning. Meanwhile, in the case of drawing, time-sharing measurements with a time interval of $40 \mathrm{~ms}$ were reported for the investigation of structure development after batch drawing of PET films ${ }^{7)-12)}$. However, the draw ratio of the study was around 4.0, which is the minimum draw ratio that can cause orientation-induced crystallization, and the strain rate of the study $\left(15 \mathrm{~s}^{-1}\right)$ was far smaller than that used in the actual fiber manufacturing process. This is presumably why the reported crystallization rate of $\left.40 \mathrm{~s}^{-1} 11\right)$ is far smaller than the rate of around $1000 \mathrm{~s}^{-1}$ obtained by the temperature profiles in the processes of high-speed melt spinning ${ }^{13)}$ and drawing ${ }^{33)}$. There are other reports on the continuous pin drawing process dealing with the draw ratio dependence of fiber structure 
development ${ }^{14-16)}$. Although the take-up speed in these studies reached as high as $27 \mathrm{~m} / \mathrm{min}^{15}$, the results for the first $51 \mathrm{~ms}$ after necking could not be obtained because the measuring point was fixed $23 \mathrm{~mm}$ away from the pin drawing point.

We have reported the results of in-situ measurements on the basis of the same principles as in the following studies: drawing behavior ${ }^{30)}$, fiber temperature profile ${ }^{33-34)}$ and wide-angle $\mathrm{x}$-ray diffraction (WAXD)/small-angle $\mathrm{x}$-ray scattering (SAXS) analysis ${ }^{35)}$. That is, we have obtained the data as an accurate function of elapsed time after necking by measuring the distance from the measuring point to the necking point. The necking can be fixed in space accurately without any contact by rapid and uniform laser irradiation heating of the running fiber. By comparing the temperature profiles obtained by in-situ measurement with the profiles estimated from the drawing condition, the crystallization rate $\mathrm{e}^{33)}$ and energy balance ${ }^{34)}$ in the drawing process were analyzed. As the result, part of the applied work of the drawing stress was stored as elastic energy, which was released in a few milliseconds with the progress of fiber structure development. WAXD/SAXS images were taken at a time interval of $4 \mathrm{~ms}^{35)}$. However, a time precision of $\pm 8 \mathrm{~ms}$ is not sufficient for analyzing the fiber structure development. In addition, there was data for only one drawing condition. Moreover, the $\left(001^{\prime}\right)$ diffraction, which is reported to correspond to the metastable intermediate phase $^{9), 23)-28)}$, could not be observed in our previous study ${ }^{35)}$.

In the present study, WAXD/SAXS images were taken with an ultra-high luminant synchrotron radiation beam. By using the synchrotron $\mathrm{x}$-ray source, the $\mathrm{S} / \mathrm{N}$ ratio and space precision of the images improved far beyond that in the above-mentioned report, i.e., Ref. 35. The time accuracy of the measurement improved as well, to less than $\pm 0.6 \mathrm{~ms}$. The $\mathrm{x}$-ray images were taken for the corresponding elapsed time from less than $0-320 \mathrm{~ms}$ after necking, with a time interval of $0.5 \mathrm{~ms}$ for the initial stage of fiber structure development. The dependence of the draw ratio on the fiber structure development was investigated through $\mathrm{x}$-ray images for draw ratios in the range of 4.0-6.0. In addition, temperature profiles were measured at the same draw conditions as in the case of the WAXD/SAXS measurements. 


\section{Experimental}

\subsection{Measurement Principle}

The in-situ measurement system for the laser drawing process is schematized in Figure 1. Please refer to our previous reports for details ${ }^{32-35}$. The running fiber was heated through three-directional irradiation by a $\mathrm{CO}_{2}$ laser beam generated by a PIN-20S laser source (rated power of $20 \pm 1 \mathrm{~W}$ ) manufactured by Onizuka Glass Co. Ltd. The diameter of the laser beam was $5 \mathrm{~mm}$. The fiber was drawn by the speed difference between the feed and take-up rollers. The drawing was very stable and steady because the necking was fixed within the laser irradiation range $\mathrm{e}^{32)}$. Thus, we could analyze the fiber structure for a certain elapsed time $t$ after external force deformation by taking WAXD/SAXS images and measuring the fiber temperature at position $x$, defined as the distance from the necking point shown in Figure 1. For the WAXD/SAXS measurements, $x$ was adjusted by moving the necking point, which is achieved by moving the mirror unit, and hence the laser irradiation point, along the fiber running direction. In the case of temperature measurement, $x$ was adjusted by moving the measurement point, i.e., by moving a thermometer.

\subsection{Drawing Conditions}

The monofilament used for drawing in this study was prepared by melt-spinning of PET $([\eta]=0.935$ $\mathrm{dl} / \mathrm{g}$ ) provided by Toray, Co., Ltd. The spinning temperature was $300^{\circ} \mathrm{C}$, the mass flow rate was $5.20 \mathrm{~g} / \mathrm{min}$, the take-up speed was $79 \mathrm{~m} / \mathrm{min}$, and the monofilament diameter was $248 \mu \mathrm{m}$. The drawing conditions are shown in Table 1. The monofilament was drawn 4.0-6.0 times, and taken up at 6.0 and $48 \mathrm{~m} / \mathrm{min}$. The draw ratio range is chosen so as to ensure stable neck drawing. Neither neck drawing nor orientation-induced crystallization occurs below 4.0 times, and the fiber tends to break above 6.0 times. The laser power values in Table 1 were measured with a power-meter (Synrad Co., Ltd., PW-250). Although a slight fluctuation in laser power was observed during the measurements, this would hardly influence the fiber structure development because the drawing stress was stable. 


\subsection{Temperature Measurement}

The fiber temperature was measured by an infrared thermometer manufactured by Japan Sensor Co., Ltd. $^{33,34)} \mathrm{A} \mathrm{HgCdTe}$ detector was used for the thermometer, and the response time was $10 \mathrm{~ms}$. The thermometer was equipped with an interference filter for $5.78 \mathrm{~mm}$ wavelength, which corresponds to the stretch vibration of the PET carbonyl group. Further details on in-situ fiber temperature measurement can be found in previous reports ${ }^{33-34)}$.

\section{$2.4 \quad$ X-ray Images}

The synchrotron radiation x-ray source beam line 40B2 of SPring- 8 was used for this study. The applied $\mathrm{x}$-ray wavelength was $0.10 \mathrm{~nm}$. The diameter of the $\mathrm{x}$-ray beam was about $0.2 \mathrm{~mm}$. WAXD/SAXS images were taken by a $3000 \times 3000$ pixel imaging plate with 400 - and 1000 -mm-long vacuum chambers. The exposure times of the WAXD and SAXS images were $180 \mathrm{~s}$ and $300 \mathrm{~s}$, respectively. The camera lengths were calibrated by the diffractions of lead stearate $(\mathrm{StPb})$. The neck-drawing position was determined by the transmitted x-ray intensity profile obtained by the ion chamber. Diffractions that were due to the polyimide film mounted on the vacuum chamber ${ }^{25)}$ were observed in the WAXD/SAXS images obtained. These were used as reference when compensating for the background scattering by subtracting a blank image. Further details on in-situ WAXD/SAXS measurement are presented in our previous report ${ }^{35)}$.

The intensity profiles along the diffraction angle were taken from the obtained images. The d-spacing and size of the fiber structure were estimated from the diffraction peaks of the profiles. The position $\phi_{0}$ and full width at half maximum $\sigma$ of the diffractions were determined by fitting a Gaussian function (Eq. 1). The beam divergence effect on $\sigma$ was corrected by the width of the intensity profiles for $\mathrm{StPb}$. The spacing $d$, structure size $D$, and distortion of the second kind $g_{I I}$ were estimated by Bragg's equation (Eq. 2), the Hosemann plot (Eq. 3), and Scherer's equation (Eq. 4), where the constant $k$ in Eq. 4 was 0.918 . The crystal orientation factor $f$ was estimated from the azimuthal intensity profiles for the $(010),(\overline{1} 10)$ and (100) planes. For this purpose, azimuthal intensity profiles were fitted by a Pearson VII equation (Eq. 5) ${ }^{30)}$, assuming equal width $\tau$ for the three diffractions. The crystal orientation $f$ was estimated using Eq. 6 by integrating the fitted 
profiles.

$$
I(\phi)=I_{0} \exp \left\{-4 \ln 2 \cdot\left(\frac{\theta-\theta_{0}}{\sigma}\right)^{2}\right\}
$$

$$
\begin{aligned}
& \left(\frac{\sigma_{m} \cos \theta_{0 m}}{\lambda}\right)^{2}=\left(\frac{1}{D}\right)^{2}+\frac{\left(\pi g_{I I} m\right)^{4}}{d_{1}^{2}} \\
& D=\frac{k \lambda}{\sigma \cos \theta_{0}}
\end{aligned}
$$

$I(\beta)=-\frac{I_{0}}{\left\{1+4\left(\frac{\beta-\beta_{0}}{\tau}\right)^{2}\left(2^{\frac{1}{2.5}}-1\right)\right\}^{2.5}}$

$$
f=\frac{3\left\langle\cos ^{2} \beta\right\rangle-1}{2},\left\langle\cos ^{2} \beta\right\rangle=\frac{\int_{0}^{\pi / 2} I(\beta) \cos ^{2} \beta \sin \beta d \beta}{\int_{0}^{\pi / 2} I(\beta) \sin \beta d \beta}
$$

Eq. 6

\section{Results and Discussion}

\subsection{Temperature Profiles}

Temperature profiles along the fiber are shown in Figure 2. The horizontal axes of (a) and (b) represent the distance from the laser beam axis along the running fiber direction, while those of $(c)$ and (d) represent the elapsed time after necking. As shown in Figs. 2(a) and (b), the laser-heated fiber deforms abruptly at about $1.0 \mathrm{~mm}$ before the laser beam axis. At the necking point, the fiber temperature rises instantaneously with the plastic deformation. The fiber temperature just before the necking point decreases with increasing draw ratio. These results agree with the results of Okumura et al. ${ }^{32-33)}$, who reported ${ }^{33)}$ that the hot-drawing form transformed into the cold-transformed form at a pre-drawing temperature around the glass-transition temperature of $80^{\circ} \mathrm{C}$. As shown in Fig. 2(c), this condition holds true for draw ratios in the 4.5 and 5.0 range in the present study, and the drawing stress increases steeply above this range. Thus, we regard the drawing 
form in this study as hot-drawing for draw ratios below 4.5, and cold-drawing above draw ratios of 5.0.

Under all drawing conditions, the fiber temperature attains its maximum value at about $2.5 \mathrm{~mm}$ past the laser beam axis, where the fiber passed through the laser beam. The maximum temperature increases with increasing draw ratio for both take-up speeds. As described later, the main features of the fiber structure emerges within $2.0 \mathrm{~ms}$ of necking, and then the structure is stabilized in several hundreds of milliseconds. As shown in Figures 2(c) and (d), under all drawing conditions, the fiber temperature stays at $130-150^{\circ} \mathrm{C}$ within the first $2.0 \mathrm{~ms}$ of necking, peaks from 8 to several tens of milliseconds around the edge of the laser beam, and then cools below the glass transition temperature in several hundreds of milliseconds.

\subsection{Transmitted X-ray Intensity}

Transmitted x-ray intensity profiles are shown in Figure 3. Because the transmitted x-ray intensity decreased by the absorption and scattering of the incident x-ray, it can be concluded that the intensity depended on the cross-sectional area of the running fiber in the x-ray irradiation area. Thus, we can determine the position and fluctuation in necking by the intensity profile. A clear increase in transmitted intensities is observed in a certain region along the fiber running direction under all drawing conditions in the figure. The center of the region of intensity variation presumably indicates the necking position. Video observation confirms that neck-drawing occurs in these regions of intensity increase. Because the elapsed time after necking $t$ is calculated from the distance between the measurement point and the necking point, which is determined by the intensity profiles in the present study, the precision of the necking position determines the precision of $t$. The precision of the necking position can be estimated by the width of the region of intensity change in Figure 3. Thus, the time precisions were estimated to be $\pm 0.31 \mathrm{~ms}$ and $\pm 1.0 \mathrm{~ms}$ from the widths $\pm 0.25 \mathrm{~mm}$ and $\pm 0.1 \mathrm{~mm}$ for the take-up speeds of $48 \mathrm{~m} / \mathrm{min}$ and $6 \mathrm{~m} / \mathrm{min}$, respectively.

The origin of the horizontal axis in Figure 3, defined as the laser beam axis, has low accuracy because it was adjusted by visual inspection ${ }^{35)}$. To eliminate the error associated with this, we calculated the elapsed time in the present study by using the distance from the necking point as determined by the transmitted $\mathrm{x}$-ray intensity profile, instead of the distance from the origin. The necking positions, as determined by the 
temperature profiles shown in Figure 2, are also plotted in Figure 3. The necking position determined by both measurements moves upstream with increasing draw ratio. The nearly $0.4-\mathrm{mm}$ shift in all necking positions in Figure 3(b) between the necking positions obtained by temperature profiles and those obtained by x-ray intensity profiles was presumably caused by the error in the origin. After compensation of this overall shift, the residual variance of the necking position between the two measurements dropped to a range of about \pm 0.2 $\mathrm{mm}$. This variation in the necking position could be due to the long-term fluctuation of the laser output ${ }^{36)}$, variation of fiber diameter, or shift in fiber running line. As mentioned above, from the fluctuation and variation of the necking position, the total fluctuation of the necking position can be estimated not to exceed $\pm 0.5 \mathrm{~mm}$ for a take-up speed of $48 \mathrm{~m} / \mathrm{min}$ and $\pm 0.3 \mathrm{~mm}$ for a take-up speed of $6 \mathrm{~m} / \mathrm{min}$. The resulting errors in elapsed time after necking were not larger than $\pm 0.6 \mathrm{~ms}$ and $2.8 \mathrm{~ms}$, respectively. The accuracy can be improved in the future by monitoring the necking point during x-ray imaging. In addition, because the fluctuation range of the necking position depends on the laser beam diameter, rather than the drawing speed, it is possible to analyze the structure development process at the higher drawing speed with a time error of only a few microseconds.

The time error obtained is about $4 \%$ of the time error reported in our previous study ${ }^{35)}$. It is the shortest time error reported for in-situ x-ray analysis of the fiber drawing process ${ }^{35)}$. Moreover, it is almost equivalent to the shortest time error reported for the high-speed melt spinning process ${ }^{18,19)}$. The drawing process is advantageous over the melt spinning process in that it yields clear and highly reproducible diffraction images owing to the stable and steady drawing process, in which the applied stress is higher than in spinning. In addition, the structure development before neck-drawing can be ignored in the drawing process because the fiber hardly deforms before neck-drawing. This is a great advantage over melt spinning, where the structure development before neck-like deformation is complex ${ }^{22)}$.

\subsection{X-ray Patterns}

WAXD and SAXS images are shown in Figures 4 and 6, respectively. Figure 7 shows the enlarged WAXD image for a draw ratio of 5.5 at an elapsed time of $0.5 \mathrm{~ms}$ after necking. Equatorial (a), (c) and 
meridional (b) intensity profiles taken from the WAXD images are shown in Figure 5. It is confirmed that the same amorphous halo observed for the as-spun fiber was observed for all WAXD images before necking. It can be shown for all drawing conditions that the crystal diffractions and the long period scattering appear at an elapsed time of about $1.0 \mathrm{~ms}$ and the fundamental fiber structure is formed within $1.0-4.0 \mathrm{~ms}$ of necking. Thus, we will discuss the fiber structure development process in the following three stages: the two-dimensionally ordered structure observed $0.5-1.0 \mathrm{~ms}$ after necking, the crystallization occurring 1.0-4.0 ms after necking, and the subsequent structural development.

\subsection{Two-dimensionally Ordered Fibrillar Structure}

WAXD images for $0.5 \mathrm{~ms}$ after necking are quite different from those for both before necking and 1.5 ms after necking. The images show sharp, streak-like meridional diffraction peaks, and the amorphous halo concentrated along the equatorial direction is sharper than that of the as-spun fiber. This clearly indicates that an intermediate metastable structure is formed during the process of fiber structure development from an oriented amorphous phase. Taking into consideration the time accuracy mentioned above, the elapsed time corresponding to these images is $0.5 \pm 0.6 \mathrm{~ms}$ after necking, which guarantees that the structure corresponds to the images formed less than $1.0 \mathrm{~ms}$ after necking. This means that the two-dimensionally ordered structure that emerged immediately after necking has high regularity. In addition, the streak-like shape of the meridional diffractions also indicates that the two-dimensionally ordered structure has a long fibrillar shape along the fiber axis. Because the structure has a two-dimensional regularity, the normal to the benzene rings lies in the plane perpendicular to the fiber axis, but does not align in any particular direction in this plane. Thus, no sheeting develops in the structure, while random intermolecular interactions between the carbonyl dipoles $^{38)}$ seem to result in a periodic positional regularity along the fiber axis.

The lowest-angle meridional diffraction in Figure 7 is presumably the $\left(001^{\prime}\right)$ diffraction that has been observed in the batch-drawing of PET before the appearance of crystal diffraction ${ }^{9,23-28)}$. Although not mentioned in the report, an intensity peak corresponding to the (001') diffraction was also observed in the

WAXD profile at about $4 \mathrm{~ms}$ after neck-like deformation in the case of melt spinning ${ }^{19)}$. Streak-like 
meridional diffractions that may correspond to $\left(002^{\prime}\right)$ and $\left(003^{\prime}\right)$ are also observed in the figure. As reported previously $\left.{ }^{83)} 23\right)$,24), 28 , the two-dimensionally ordered structure is presumably a precursor of a crystal because the $\left(001^{\prime}\right)$ diffraction disappeared with the appearance of crystal diffraction. The d-spacing of the (001') plane, twice that of the (002') plane spacing, and three times that of the third layer streak-like diffraction are shown in Figure 8. In all cases, the d-spacing of the (001') and (002') planes decreased with elapsed time, while those of the third layer showed an increasing trend. Thus, the third layer streak did not seem to be the (003') diffraction of the two-dimensionally ordered fibrillar structure. Rather, it may correspond to the third layer diffraction of the disordered crystal because the spacing after crystallization is coincident with the spacing of $\left(001^{\prime}\right)$

The d-spacing of the (001') plane increased with increasing draw ratio. The d-spacing for $0.5 \mathrm{~ms}$ after necking, namely $1.064-1.068 \mathrm{~nm}$, is slightly shorter than the reported c-axis length of the PET crystal (1.075 $\mathrm{nm})^{1)}$, but is clearly longer than the $1.03 \mathrm{~nm}$ obtained for batch drawing by Ran et $\mathrm{al}^{24)}$. Ran et al. proposed the smectic $\mathrm{C}$ phase whose chain axis tilted $15.7^{\circ}$ from the fiber axis, while Kawakami et al. ${ }^{28)}$ proposed, on the basis of the change in the d-spacing values, that the smectic $\mathrm{C}$ phase transformed into smectic $\mathrm{A}$ at the boundary of the natural drawing region and stress-hardening region. According to the d-spacing value mentioned above, the two-dimensionally ordered structure observed in the present study must be the reported smectic A phase. The difference in phase can be explained by the difference in stress because the drawing stress applied in the present study is presumably higher than the natural drawing stress in the batch drawing process.

The length of the two-dimensionally ordered structure along the fiber axis estimated by the width of diffractions by Sherrer's formula (Eq. 4) is shown in Figure 9. After the onset of crystallization, a clear decrease is observed. This is presumably caused by the disruption of the structure by the crystal developed. The Hosemann's plot for (001'), (002') and the third layer diffractions are shown in Figure 10. Because the intensity of the $\left(002^{\prime}\right)$ diffraction is weaker than that of $\left(001^{\prime}\right)$, the data plotted is limited to ensure the reliability of the width. It is clear that the three plots do not lie on a line, which suggests that the third layer 
diffraction is not the (003') diffraction of the two-dimensionally ordered structure. Thus, we have estimated the lengths of the structure and its second kind distortion coefficient $g_{I I}$ from the width of (001') and (002') planes. The estimated lengths and $g_{I I}$ at $0.5 \mathrm{~ms}$ after necking are $60-70 \mathrm{~nm}$ and $3-4 \%$, respectively. The lengths correspond to 4-6 times of the long period, one-sixth of the weight-averaged PET molecular chain length, and could correspond to the distance between chain entanglements. It suggests that the two-dimensionally ordered structure may be composed of a bundle of extended chains that bear the drawing tension at the necking deformation.

\subsection{Crystallization}

Between 1.0 to $2.0 \mathrm{~ms}$ after necking, crystal diffractions appeared, gradually becoming more distinct, while the equatorial halo and the $\left(001^{\prime}\right)$ and $\left(002^{\prime}\right)$ diffractions disappeared. At the same time, the scattering intensity corresponding to the long period increased with the disappearance of the (001') diffraction in the SAXS patterns. The fact that these changes occurred simultaneously indicates that the fundamental fiber structure was formed at this time by transformation of the two-dimensionally ordered structure into a three-dimensional crystal alternated with amorphous phase.

To estimate the progress of crystallization, the crystal phase fraction was calculated from the equatorial WAXD intensity profiles with peak separation of the crystal and amorphous regions. The crystallinity index obtained is shown in Figure 11. For all draw ratios, the crystal fraction increased to a half of that of the drawn fiber obtained at $2.0 \mathrm{~ms}$ after necking, reaching $80 \%$ at $10 \mathrm{~ms}$ after necking, and continued to increase gradually after that. We think that the increase in crystal diffraction intensity after $10 \mathrm{~ms}$ was not caused by the increase in the amount of crystal, but rather, mainly by the increase in crystallinity, as shown in Section 3.6. Thus, we estimated the crystallization rate from the crystallinity index $\chi$ for an elapsed time of less than 5 ms. The derived Avrami equation (Eq. 8) for one-dimensional heterogeneous nucleation was adopted for the calculation: 


$$
\chi=\left[1-\exp \left\{-K\left(t-t_{0}\right)\right\}\right] \chi_{\infty} \quad \text { Eq. } 8
$$

where the crystallization rate $K=700 \mathrm{~s}^{-1}, \chi_{\infty}=0.45$, and $t_{0}=0.6 \mathrm{~s}$ was determined by curve fitting. The delay time $t_{0}$ may correspond to the formation time of the two-dimensional ordered structure.

The fiber temperature, calculated from the obtained crystallization rate using the energy balance equation (Eq. 9) ${ }^{34)}$, is shown in Figures 2(c) and (d) for a comparison with the measured temperature profiles.

$$
\Delta T=\frac{1}{W C_{p}}\left\{I(x) D_{f} A\left(D_{f}\right) \Delta x-\pi D_{f} h\left(T-T_{a}\right)+F \Delta V\right\}+\frac{\Delta H_{c}}{C_{p}} \Delta \chi \quad \text { Eq. } 9
$$

where $W, C_{p}$ and $D_{f}$ are the mass flow rate, specific heat, and fiber diameter, respectively. The first term, pertaining to the heating by $\mathrm{CO}_{2}$ laser irradiation, was estimated by the intensity profile of the laser beam $I(x)$ and the $\mathrm{CO}_{2}$ laser absorbance of PET $A$. The second term, which represents the cooling effect of the surrounding air, was estimated by the fiber temperature $T$, room temperature $T_{a}$, and heat transfer coefficient $h$, in which the heat transfer coefficient was calculated by the formula reported by Kase et $\mathrm{al}^{31)}$. The third term, resulting from the exothermic plastic deformation, was estimated by the drawing force $F$ and fiber velocity $v$ under the assumption that the whole applied work was used instantaneously for the temperature rise at the necking point. The fourth term, expressing the exothermic heat of crystallization, was estimated by the heat of fusion for a perfect crystal $H_{c}^{30)}$ and the crystallinity of the drawn fiber $\chi_{\text {DSC }}$. The $\chi_{\text {DSC }}$ values, corresponding to a temperature rise of 32-34 K, are measured by DSC and listed in Table 1 .

As shown in Figure 2, all temperatures readings taken just after necking are lower than the corresponding estimated temperatures under all drawing conditions. This agrees with one of our previous reports ${ }^{34)}$, which states that a part of the applied work is stored as elastic energy. The fiber temperature rises with the release of both stored elastic energy and latent heat of crystallization. As can be seen in the temperature rise in Figure 2(a), the rate of energy release almost agrees with the crystallization rate obtained from the x-ray results. 
Our previous manuscript on the same principle reports the onset of crystallization as $20 \mathrm{~ms}$ after necking ${ }^{35)}$. However, the time error in that study is one order of magnitude larger than in this study, and it may be larger still because of the fluctuation of the necking point in the present study. Thus, the onset time of crystallization in the present study is much more reliable. Kolb et al. reported that crystallization was completed within $20 \mathrm{~ms}$ in the case of the $4200-\mathrm{m} / \mathrm{min}$ melt spinning-line ${ }^{19)}$, while the degree of crystallization reported in the manuscript appears to be almost saturated within 4 ms of necking. The latter result agrees with the crystallization rate estimated by the temperature profile for $6000-\mathrm{m} / \mathrm{min}$ melt spinning ${ }^{13)}$ as well as with the results of the present study. Thus, we believe that there is no fundamental difference between the fiber structure development rates of PET in the processes of high-speed melt spinning and drawing, while there is a slight difference in the time dependence of the crystal orientation factor, as discussed below.

\subsection{Crystal Size and Orientation}

Although the fundamental fiber structure is formed within $2.0 \mathrm{~ms}$ of necking, the packing and orientation of the crystal formed continue to improve until solidification.

The d-spacings estimated from four diffractions are shown in Figure 12. The d-spacing for the equatorial diffractions show a clear decrease from about $20 \mathrm{~ms}$ after necking, in which the maximum decrease is observed for the (100) plane, and the minimum decrease is observed for the (010) plane, while the d-spacing for the ( $\overline{1} 03$ ) plane slightly increases. The crystal density increases by about $5 \%$, which can be interpreted as the thermal shrinkage of the crystal upon cooling from the maximum temperature of about $200^{\circ} \mathrm{C}$ to room temperature ${ }^{37)}$. Even for the drawn fiber, $d$-spacing for each plane is $1-2 \%$ larger than the value calculated from reported crystal parameters for drawn and annealed film ${ }^{1)}$. It is presumably caused by less crystal perfection and/or the camera length error caused by d-spacing error of lead stearate.

The crystallite sizes estimated for the diffractions are shown in Figure 13. These values show almost no time dependence except for a slight increasing trend in the case of the (100) direction. This means that the crystal that formed within several milliseconds of necking stopped growing after that, which suggests that 
almost no lamellar crystal is formed in this case. Moreover, the crystallite sizes perpendicular to the chain axis are almost the same as the diameter of the two-dimensionally ordered structure, which is presumably because almost all of the crystals were formed by transformation of the two-dimensionally ordered structure.

The crystal orientation factor is shown in Figure 14. It increases with increasing draw ratio and elapsed time after necking. The increase with time occurs simultaneously with the improvement in chain packing, which shows the rearrangement of the fiber structure with high-temperature annealing. By contrast, the crystal orientation factor ${ }^{19)}$ reported for the high-speed melt spinning process did not change along the spinning line. The difference could be due to the difference in applied stress, which is deduced by the lower reported crystal orientation value of 0.9 . It could also be caused by the difference in temperature profile after crystallization, which decreased monotonically for spinning, but increased up to about $200^{\circ} \mathrm{C}$ for drawing. Moreover, the tilting of the c-axis of the unit cell is shown in Figure 4. In particular, at a draw ratio of 4.0, the (010) and (100) diffractions were $0-4^{\circ}$ off the equator, while the ( $\left.\overline{1} 10\right)$ diffraction was almost on the equator. The separation clearly decreased with increasing draw ratio, but showed no time dependence.

The long periods obtained by the SAXS images are shown in Figure 15. The figure shows the decreasing trend before an elapsed time of $10 \mathrm{~ms}$, which corresponded to the time of crystal formation. The formation of the additional crystallites in the microfibril presumably shortens the interval between the crystallites.

\subsection{Overview of Fiber Structure Development}

The fiber structure development in the neck-drawing process of PET is schematized in Figure 16. A one-dimensionally ordered nematic-like structure (Figure 16(a)) presumably forms just after necking deformation of the amorphous fiber. The two-dimensionally ordered fibrillar structure shown in Figure 16(b) is formed from the one-dimensionally ordered structure in less than $1 \mathrm{~ms}$. The intermolecular interactions of carbonyl dipoles presumably act as the driving force for this transformation. A three-dimensionally ordered triclinic crystal (c) is developed from the two-dimensionally ordered structure in a few milliseconds. The transformation from the two-dimensionally ordered structure into the crystal would be initiated by the sheeting induced by the alignment of the carbonyl dipole direction ${ }^{38)}$. Following that, the sheets shift each 
other to avoid the benzene ring stacking with the tilt and slightly extend their chains. The two-dimensionally ordered fibrillar structure vanished with the formation of the long period structure (e). It suggests that the two-dimensionally ordered structure transformed to the so-called microfibril. In several hundreds of milliseconds, the eventual structure is stabilized through improvement in molecular packing of the crystal, in particular along the normal to the benzene ring, and through the structure rearrangement that occurs as a result of the slight increase in crystal orientation.

The fiber structure development shows no obvious draw ratio dependence with the exception of the (001') spacing and crystal orientation factor. In the temperature measurement discussed above, we assumed the drawing form in this study as hot-drawing for draw ratios below 4.5 , and cold-drawing above draw ratios of 5.0. But the difference in drawing form does not affect the fiber structure development very much. We think that this can be explained by the fiber temperature. While hot- and cold-drawing differ in the fiber temperature before necking, the temperature during fiber structure development does not vary appreciably.

The results obtained in the present study help elucidate fiber structure development in the continuous high-stress drawing of PET, in particular the two-dimensionally ordered structure formed in the initial stage of structure development and the transformation of this structure into a triclinic crystal. But the time precision of $\pm 600 \mu$ s obtained in this study was not sufficient for analyzing the structural transformation from the one-dimensionally ordered structure to the two-dimensionally ordered structure. However, adapting this principle to the high-speed drawing process will reduce the time precision to several microseconds, which may enable the structural transformation to be analyzed.

\section{Acknowledgements}

The main part of this research was conducted in the large synchrotron radiation facility SPring-8 (2005A and 2005B). We are pleased to acknowledge the support of Dr. Sono Sasaki with the experimentation at SPring-8. We would also like to express our gratitude to Toray Co. Ltd. for the supply of materials, and to Ms. Togari for her excellent illustration. This research was supported by a Grant-in-Aid for $21^{\text {st }}$ Center of 
Excellence (COE) Research from the Japanese Ministry of Education, Culture, Sports, Science, and Technology, and Grants-in-Aid No. 15350135, 15550182, and 18550191 from the Japan Society for the Promotion of Science.

\section{Reference}

1) Daubeny, R. P.; Bunn, C. W.; Brown, C. Proceedings of the Royal Society 1954, A 226, 531.

2) Zaukelies, D. A. Journal of Applied Physics 1962, 33, 3235.

3) Beresford, D. R.; Bevan, H. Polymer 1964, 5, 247.

4) Dismore, F.; Statton, W. O. Journal of Polymer Science Part B 1964, 2, 1113.

5) Peterlin, A.; Ingram, P.; Kiho, H. Die Makromolekulare Chemie 1965, 86, 294.

6) Somani, R. H.; Yang, L.; Zhu, L.; Hsiao, B. S. Polymer, 2005, 46, 8587.

7) Blundell, D. J.; Mackerron, D. H.; Fuller, W.; Mahendrasingam, A.; Marian, C.; Oldman, R. J.; Rule, R. J.; Riekel, C. Polymer, 1996, 37, 3303.

8) Mahendrasingam, A.; Marian, C.; Fuller, W.; Blundell, D. J.; Oldman, R. J.; Harvie, J. L.; Mackerron, D. H.; Riekel, C.; Engstrom, P. Polymer 1999, 40, 5553.

9) Mahendrasingam, A.; Martin, C.; Fuller, W.; Blundell, D. J.; Oldman, R. J.; MacKerron, D. H.; Harvie, J. L.; Riekel, C. Polymer, 2000, 41, 1217.

10) Blundell, D. J.; Mahendrasingam, A.; Marian, C.; Fuller, W.; Mackerron, D. H.; Harvie, J. L.; Oldman, R. J.; Riekel, C. Polymer, 2000, 41, 7793.

11) Mahendrasingam, A.; Blundell, D. J.; Marian, C.; Fuller, W.; Mackerron, D. H.; Harvie, J. L.; Oldman, R. J.; Riekel, C. Polymer, 2000, 41, 7803.

12) Mahendrasingam, A.; Blundell, D. J.; Wright, A. K.; Urban, V.; Narayanan, T.; Fuller, W. Polymer $2003,44,5915$.

13) Kikutani, T.; Kawahara, Y.; Matsui, T.; Takaku, A.; Shimizu, J. Seikei-Kakou 1989, 1, 333.

14) Hsiao, B. S.; Kennedy, A. D.; Leach, R. A.; Cho, B.; Harney, P. Journal of Applied Crystallography 
1997, 30, 1084.

15) Ran, S.; Zong, X.; Fang, D.; Hsiao, B. S.; Chu, B.; Ross, R. Journal of Applied Crystallography 2000, $33,1031$.

16) Wu, J.; Schults, J. M.; Samon, J. M.; Pangelinan, A. B.; Chuah, H. H. Polymer, 2001, 42, 7161.

17) Hirahata, H.; Seifert, S.; Zachamann, H. G. Polymer, 1996, 37, 5131.

18) Haberkorn, H.; Hahn, K.; Breuer, H.; Dorrer, H. D.; Matthies, P. Journal of Applied Polymer Science $1993,47,1551$.

19) Kolb, R.; Seifert, S.; Stribeck, N.; Zachmann, H. G. Polymer, 2000, 41, 2931.

20) Kolb, R.; Seifert, S.; Stribeck, N.; Zachmann, H. G. Polymer, 2000, 41, 1497.

21) Samon, J. M.; Schultz, J. M. Macromolecules, 1999, 32, 8121.

22) Kikutani, T.; Nakao, K.; Takarada, W.; Ito, H. Polymer Engineering Science 1999, 39, 2349.

23) Asano, T.; Calleja, F. J. B.; Flores, A.; Tanigaki, M.; Mina, M. F.; Sawatari, C.; Itagaki, H.; Takahashi, H.; Hatta, I. Journal of Applied Physics 2001, 90, 6006.

24) Ran, S.; Wang, Z.; Burger, C.; Chu, B.; Hsiao, B. S. Macromolecules, 2002, 35, 10102.

25) Fukao, K.; Koyama, A.; Tahara, D.; Kozono, Y.; Miyamoto, Y.; Tsurutani, N. Journal of Macromolecular Science Part B 2003, B42, 717.

26) Keum, J. K.; Kim, J.; Lee, S. M.; Song, H. H.; Son, Y. -K.; Choi, J. -I.; Im, S. S. Macromolecules, 2003, $36,9873$.

27) Keum, J. K.; Song, H. H. Polymer, 2005, 46, 939.

28) Kawakami, D.; Hsiao, B. S.; Burger, C.; Ran, S.; Avila-Orta, C.; Sics, I.; Kikutani, T.; Jacob K. I.; Chu B, Macromolecules, 2005, 38, 91 .

29) Chang, H.; Lee, K. G.; Schultz, J. M. Journal of Macromolecular Science Part B 1994, B33, 105.

30) Mehta, A.; Gaur, U.; Wunderlich, B. Journal of Polymer Science 1978, 16, 289.

31) Kase, S.; Matsuo, T. Journal of Polymer Science Part A 1965, 3, 2541.

32) Okumura, W.; Kanegae, T.; Ohkoshi, Y.; Gotoh, Y.; Nagura, M. International Polymer Processing 2003, 
$18,46$.

33) Okumura, W.; Yamaguchi, T.; Ohkoshi, Y.; Gotoh, Y.; Nagura, M. International Polymer Processing 2002, 17, 125.

34) Yamaguchi, T.; Ohkoshi, Y.; Gotoh, Y.; Nagura, M. Seikei-Kakou, 2005, 17,649.

35) Yamaguchi, T.; Komoriyama, K.; Ohkoshi, Y.; Urakawa, H.; Gotoh, Y.; Terasawa, N.; Nagura, M.; Kajiwara, K. Journal of Polymer Science Part B, 2005, 43, 1090.

36) Ikaga, T.; Kobayashi, A.; Ohkoshi, Y.; Gotoh, Y.; Nagura, M.; Urakawa, H.; Kajiwara, K. SEN’I GAKKAISHI, 2002, 58, 8.

37) Nakamae, K.; Nishino, T.; Yokoyama, F.; Matsumoto, T. Journal of Macromolecular Science Part B 1988, B27, 407.

38) Bonart, Von R. Kolloid -Zeitschrift \& Zeitschrift fuer Polymere, 1966, 213, 1. 
Table 1 Drawing Conditions

\begin{tabular}{|c|c|c|c|c|c|}
\hline \multirow{2}{*}{$\begin{array}{l}\text { Take-up } \\
\text { Speed } \\
\text { / } \mathrm{m} \mathrm{min}^{-1}\end{array}$} & \multirow{2}{*}{$\begin{array}{l}\text { Laser } \\
\text { Power } \\
\text { / W }\end{array}$} & \multirow{2}{*}{$\begin{array}{l}\text { Draw } \\
\text { Ratio }\end{array}$} & \multicolumn{2}{|c|}{$\begin{array}{c}\text { Drawing Stress } \\
\text { / MPa }\end{array}$} & \multirow{2}{*}{$\begin{array}{c}\text { Crystallinity } \\
\chi_{\text {DSC }} \\
\times 100\end{array}$} \\
\hline & & & $\begin{array}{l}\text { WAXD/SAXS } \\
\text { Measurement }\end{array}$ & $\begin{array}{l}\text { Temperature } \\
\text { Measurement }\end{array}$ & \\
\hline \multirow{5}{*}{48} & \multirow{5}{*}{$18-22$} & 4.0 & 28 & 29 & 40 \\
\hline & & 4.5 & 50 & 57 & 41 \\
\hline & & 5.0 & 78 & 85 & 43 \\
\hline & & 5.5 & 127 & 127 & 43 \\
\hline & & 6.0 & 168 & & \\
\hline \multirow{4}{*}{6.0} & \multirow{4}{*}{$3.2-3.8$} & 4.0 & 38 & 28 & 39 \\
\hline & & 4.5 & 49 & 58 & 41 \\
\hline & & 5.0 & 86 & 92 & 42 \\
\hline & & 5.5 & 122 & 120 & 42 \\
\hline
\end{tabular}




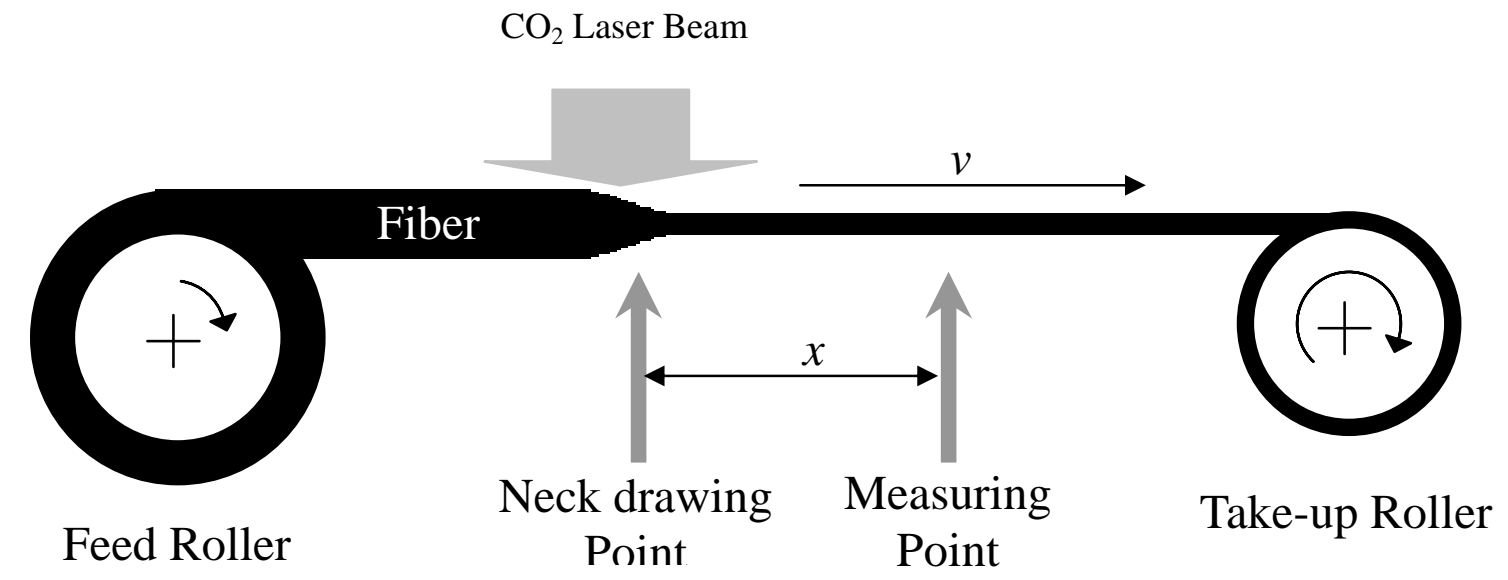

Fig. 1 


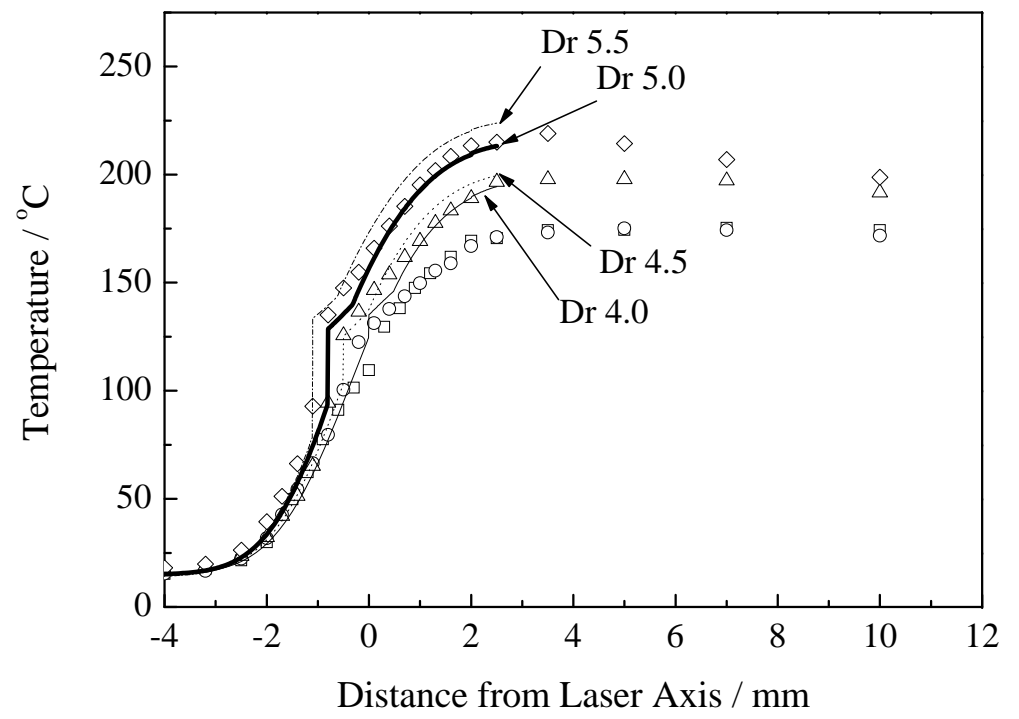

(a)

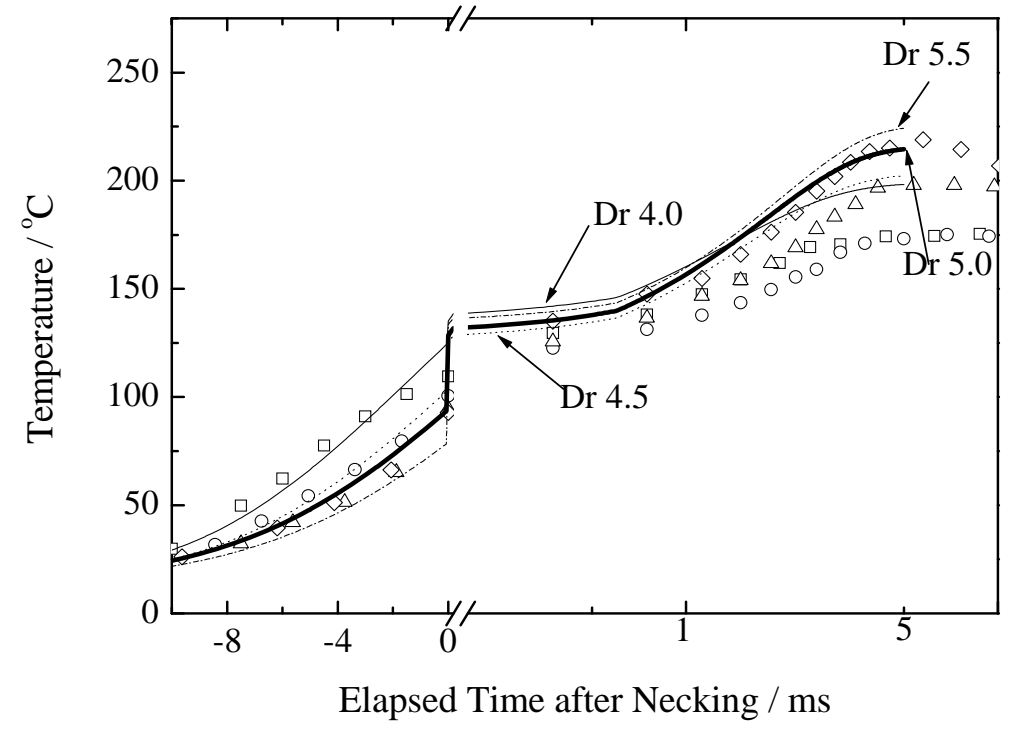

(c)

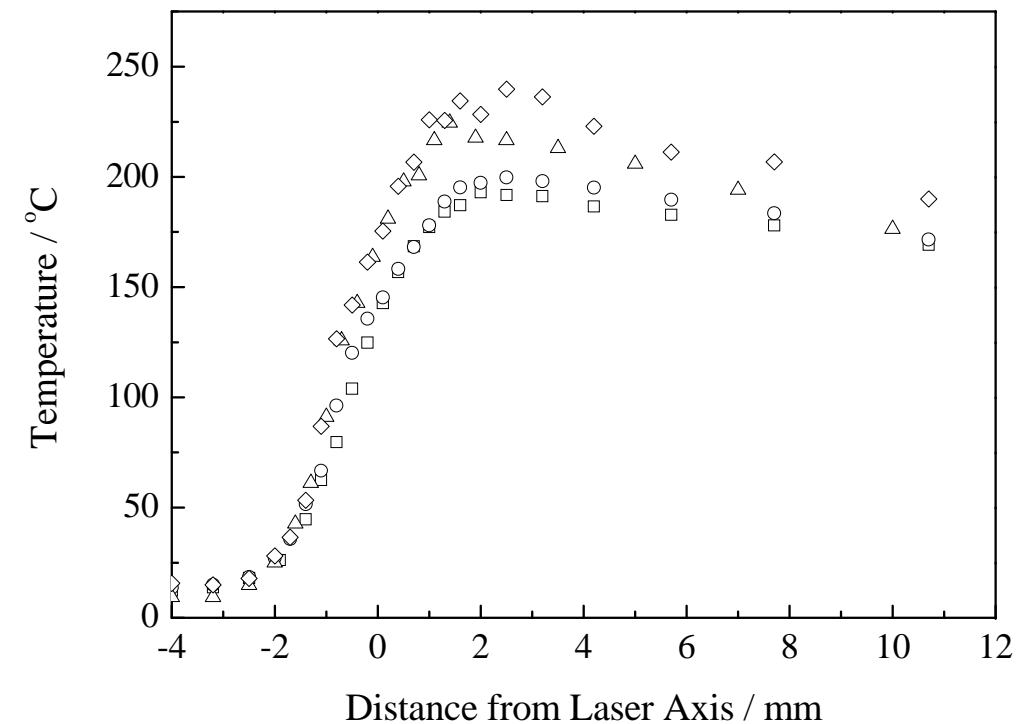

(b)

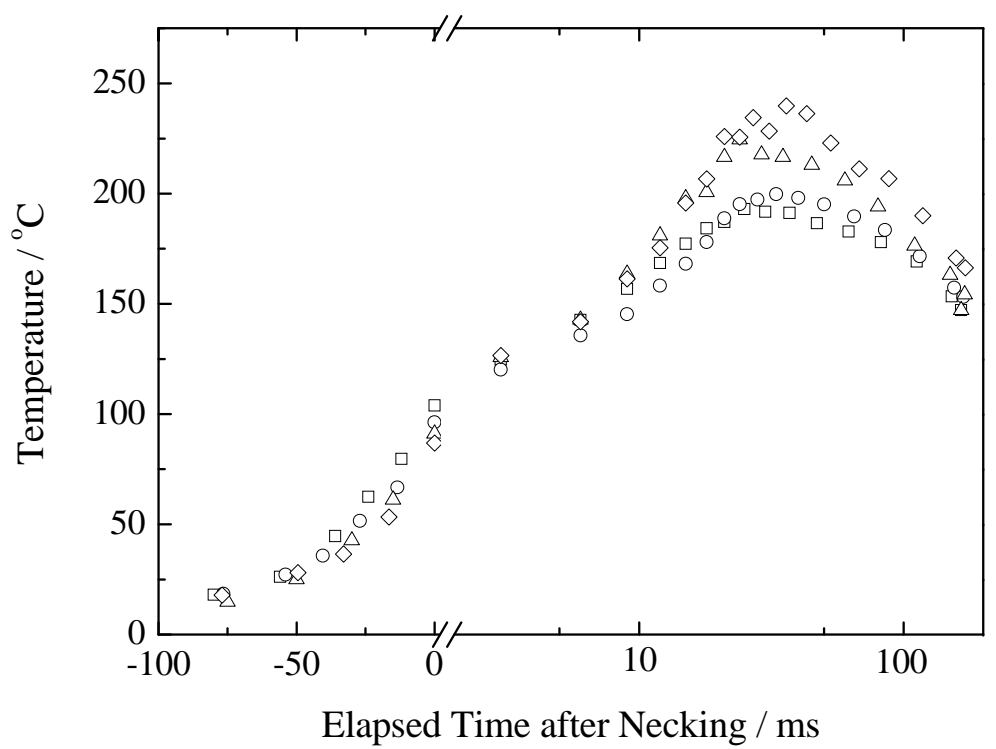

(d) 
Fig. 2 


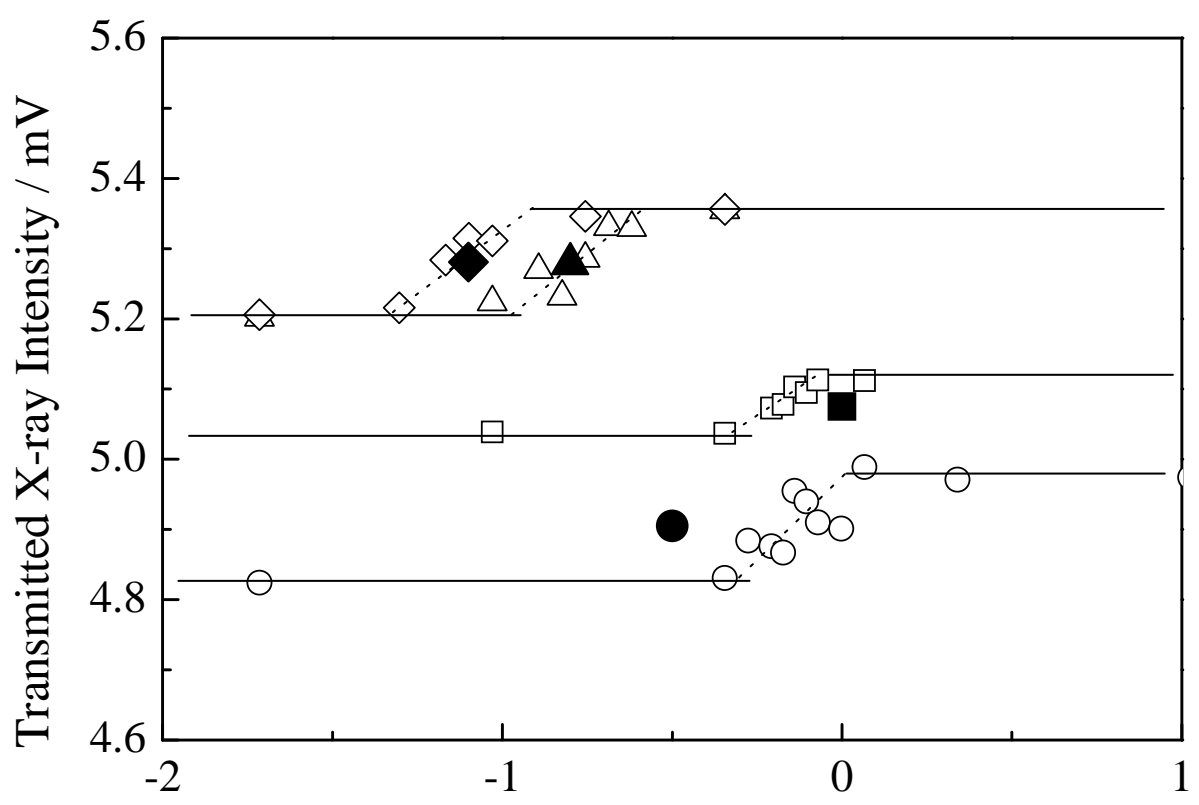

Distance from Laser Axis / mm

(a)

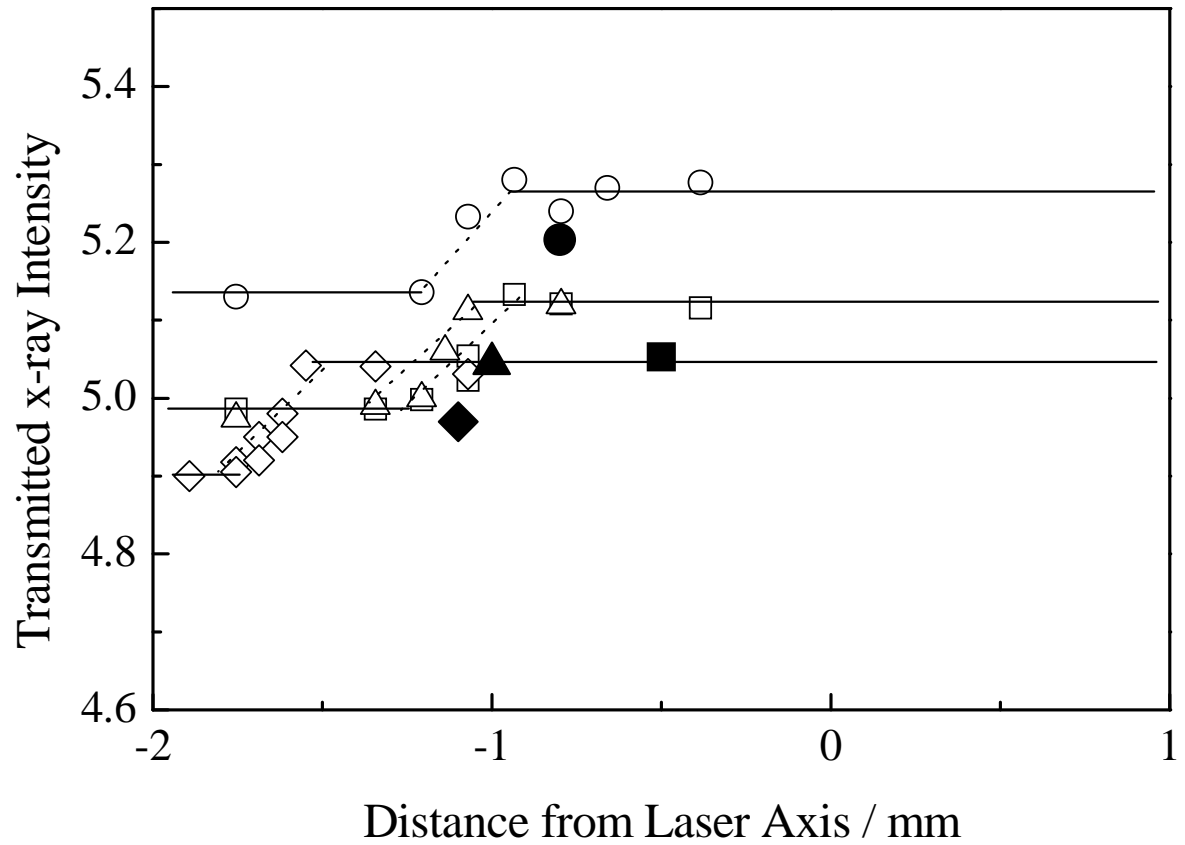

(b)

Fig. 3 


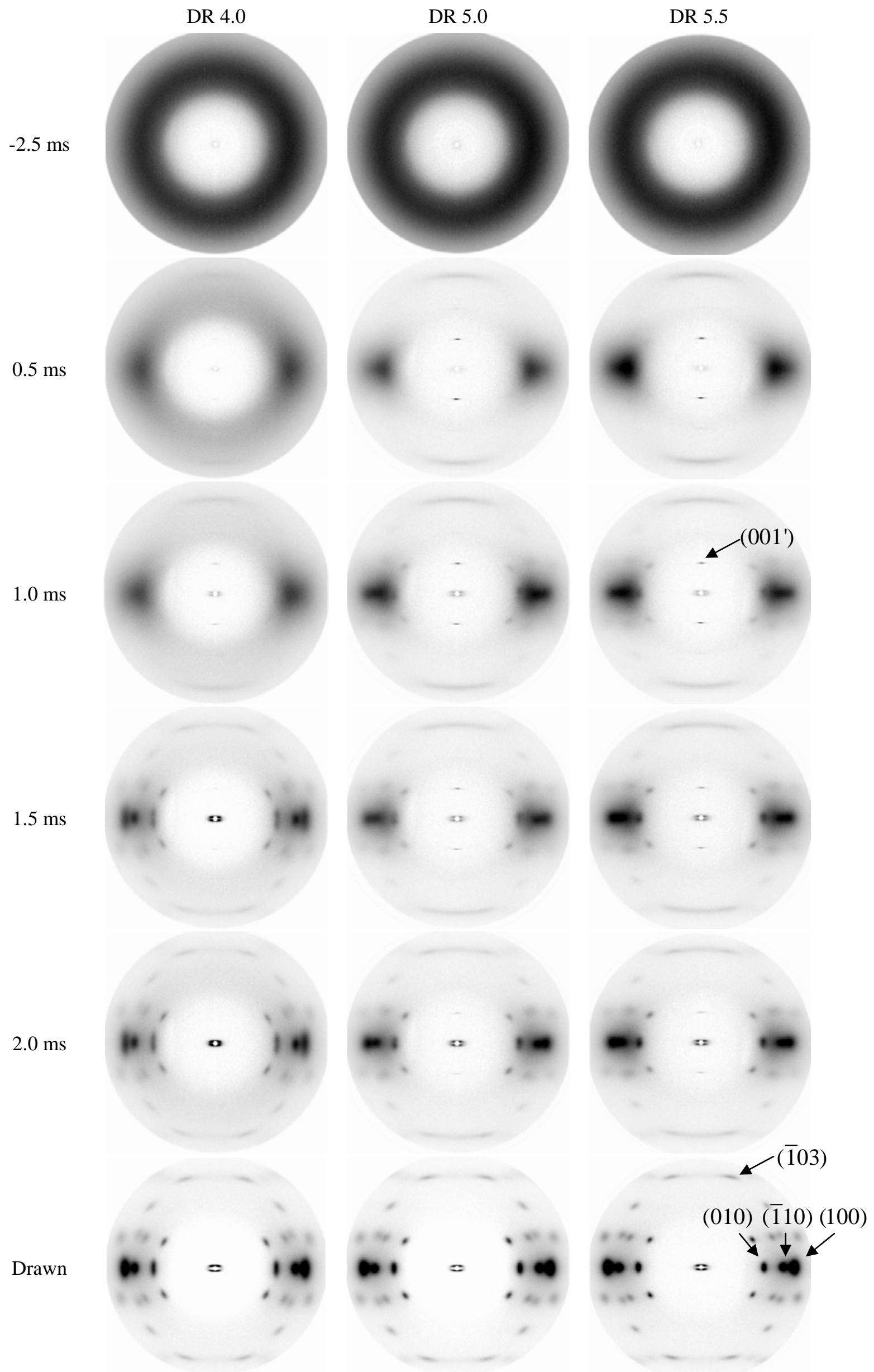

Fig. 4 

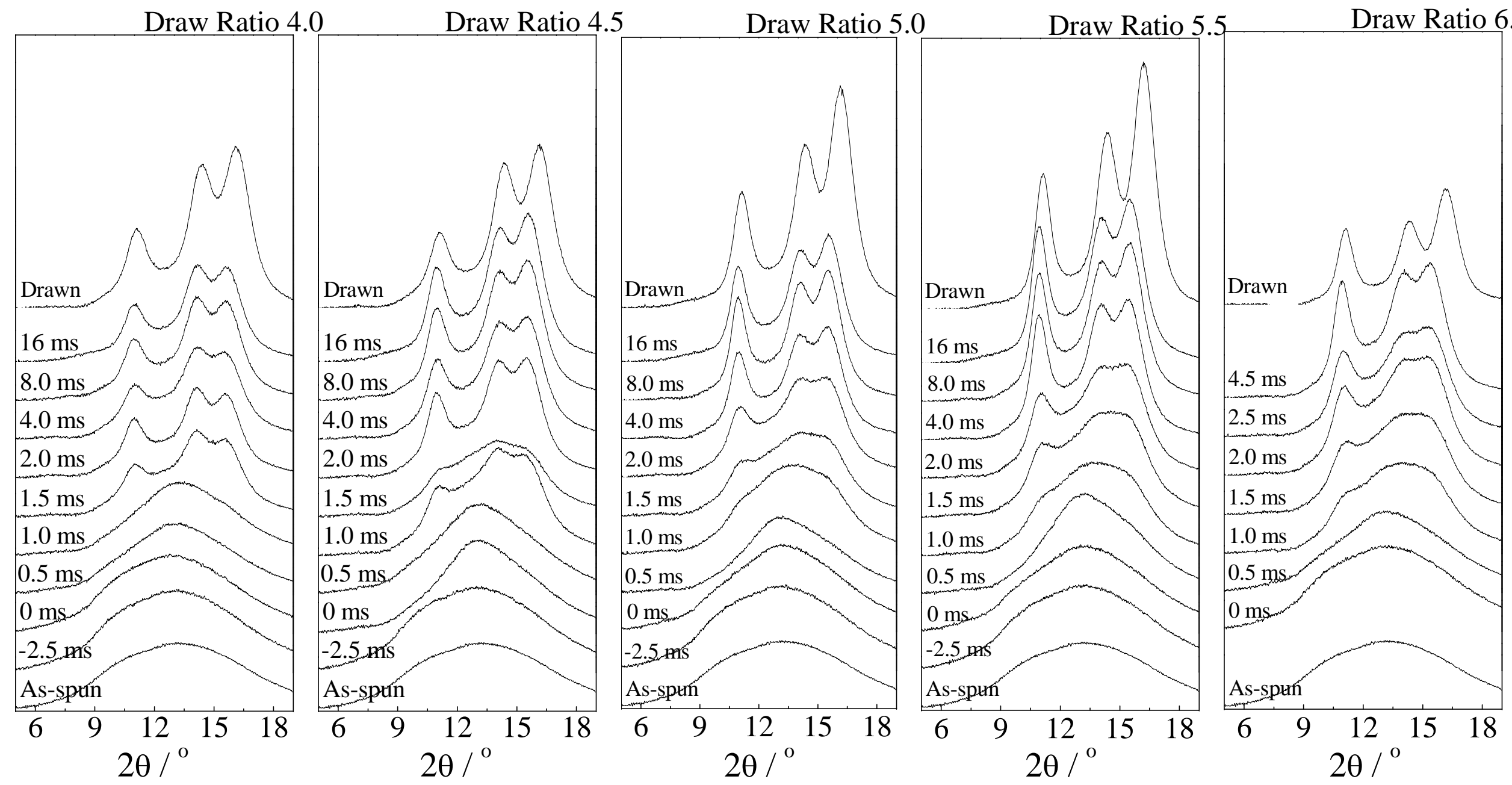

Fig. 5 (a) 

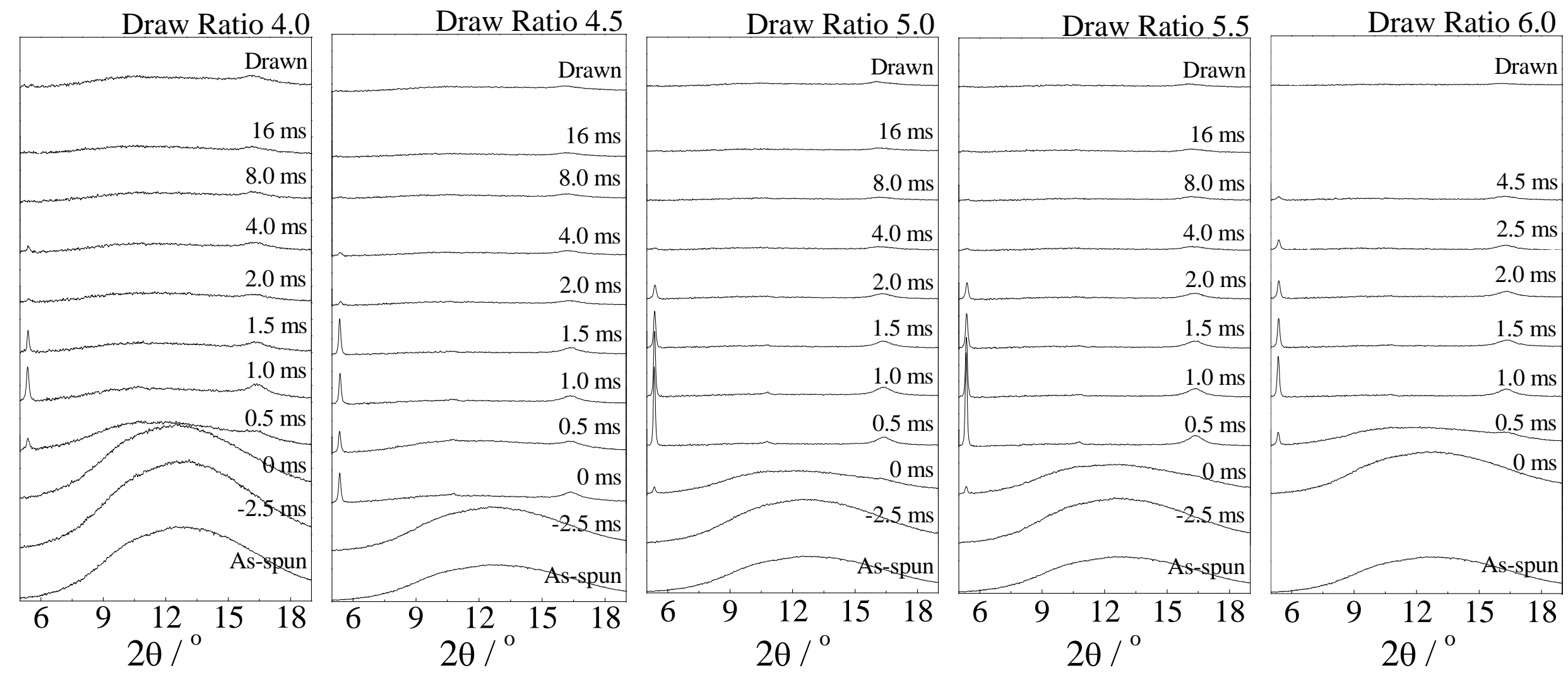

Figure 5 (b) 

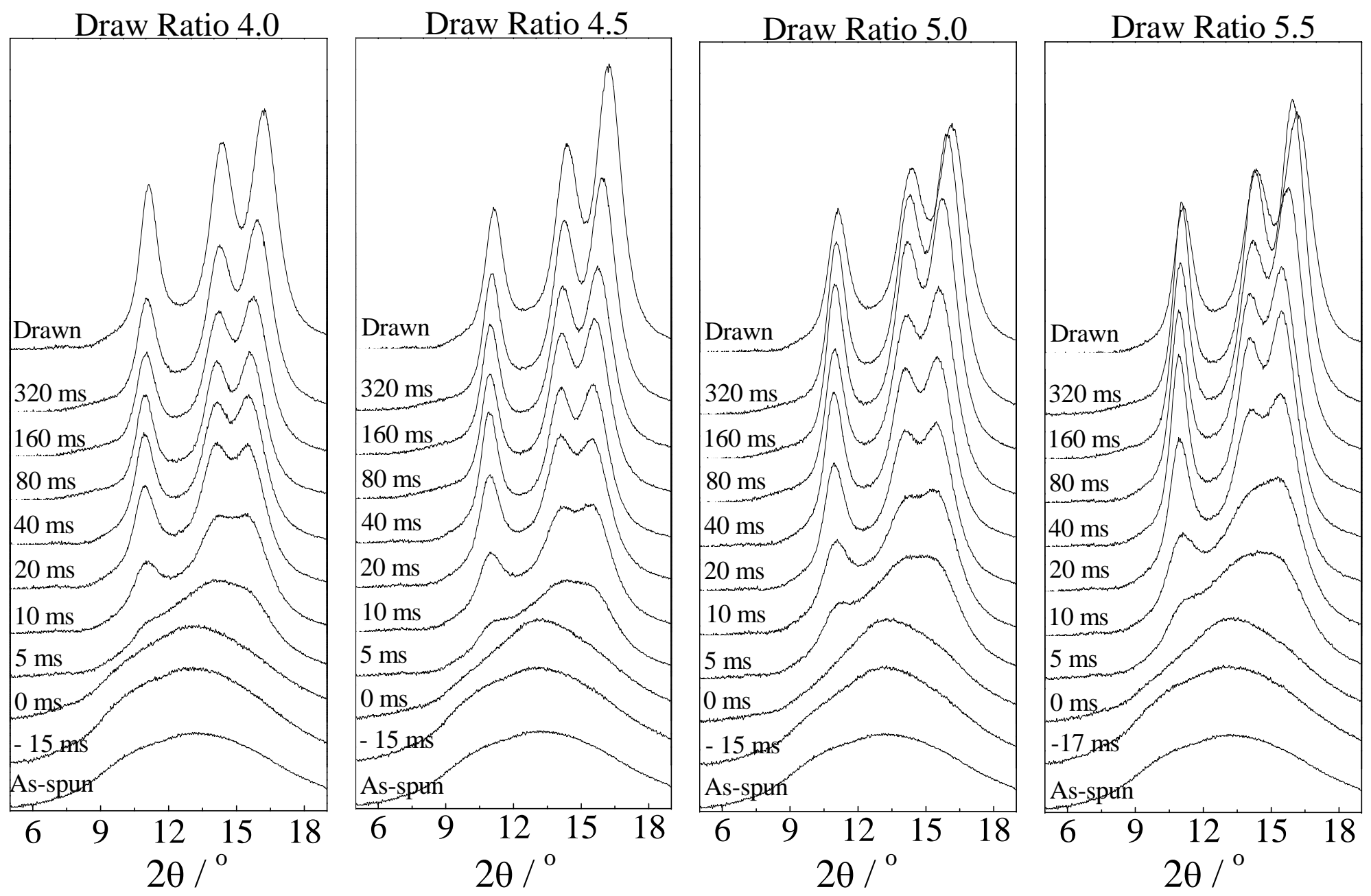

Fig. 5 (c) 


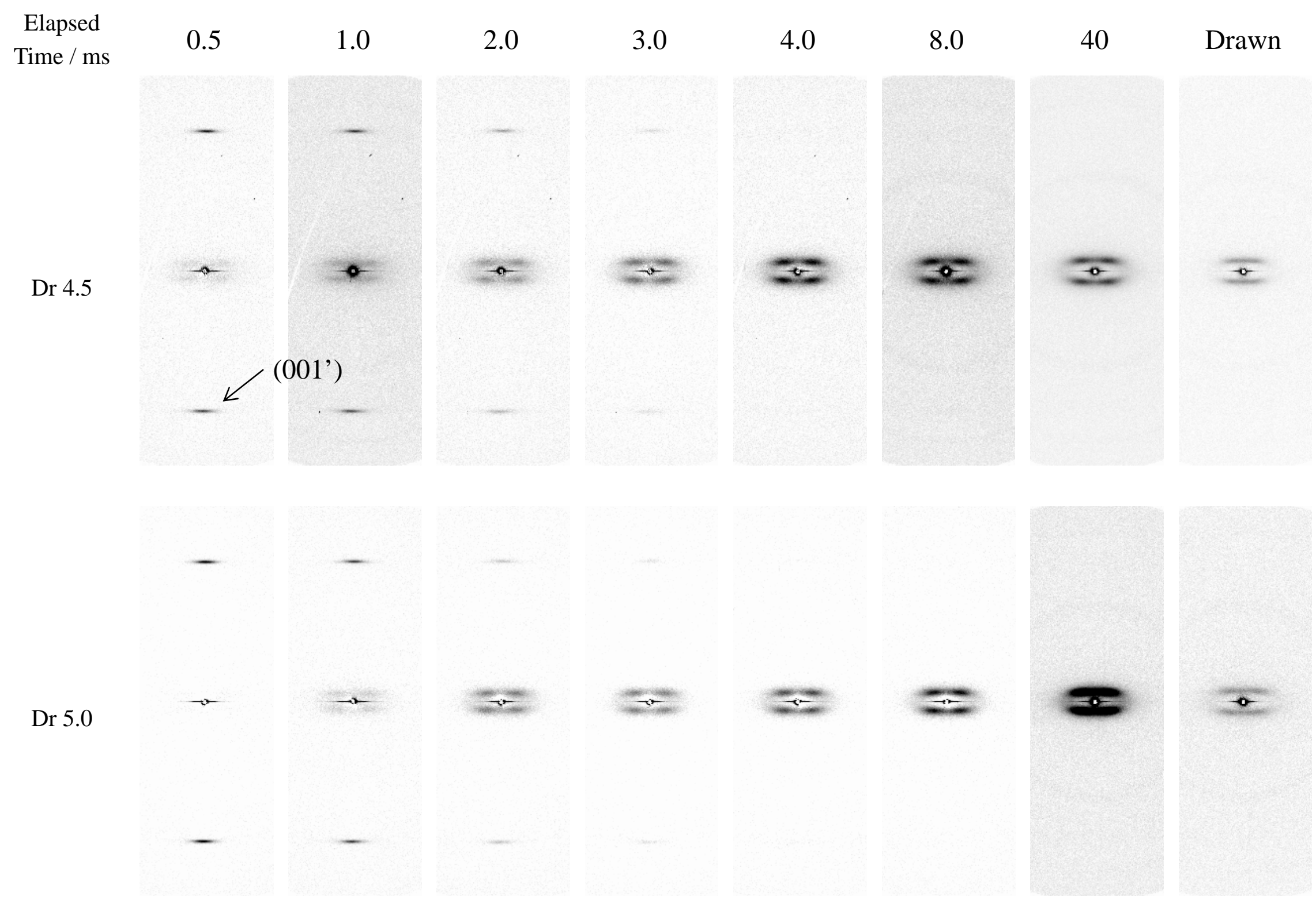

Fig. 6 


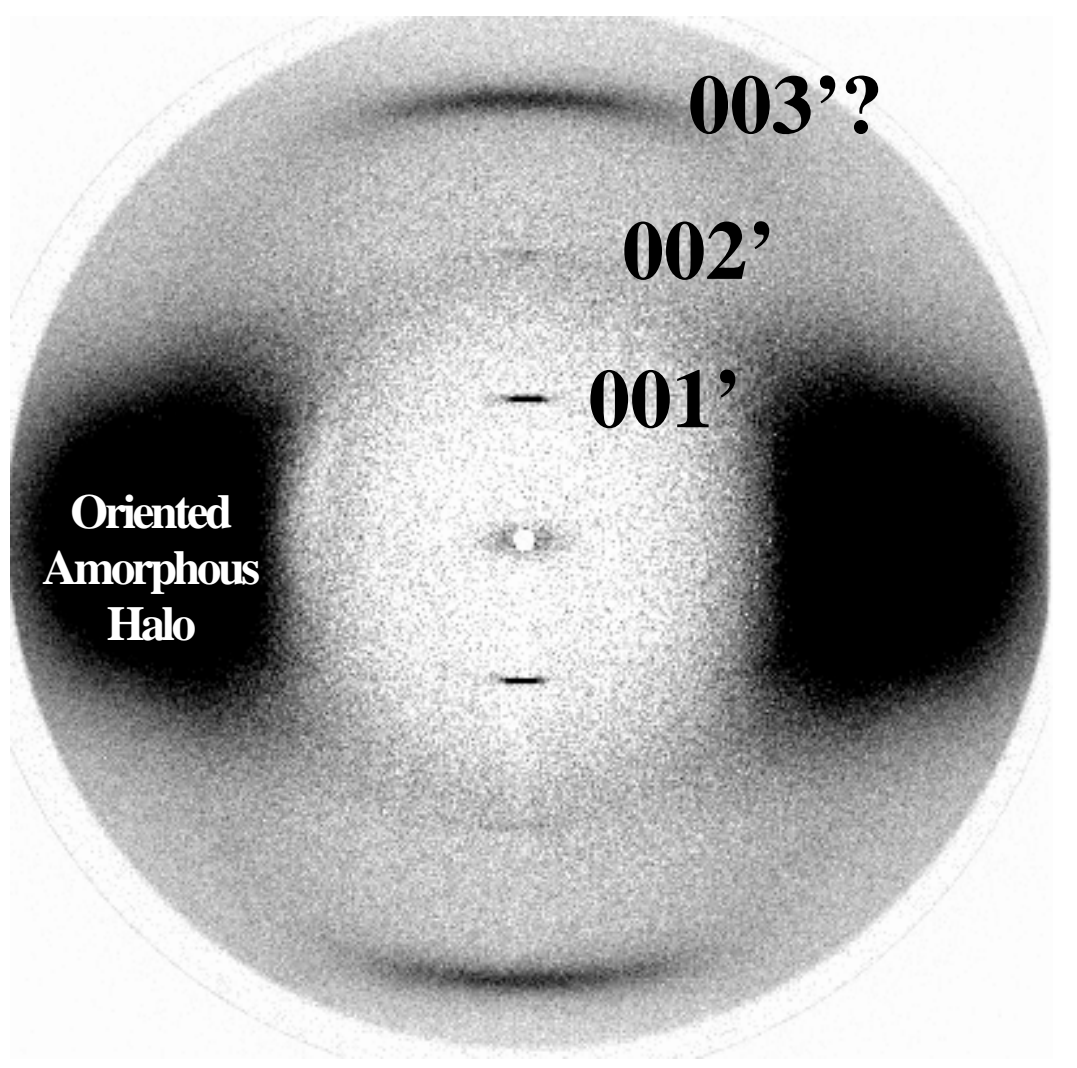

Fig. 7 


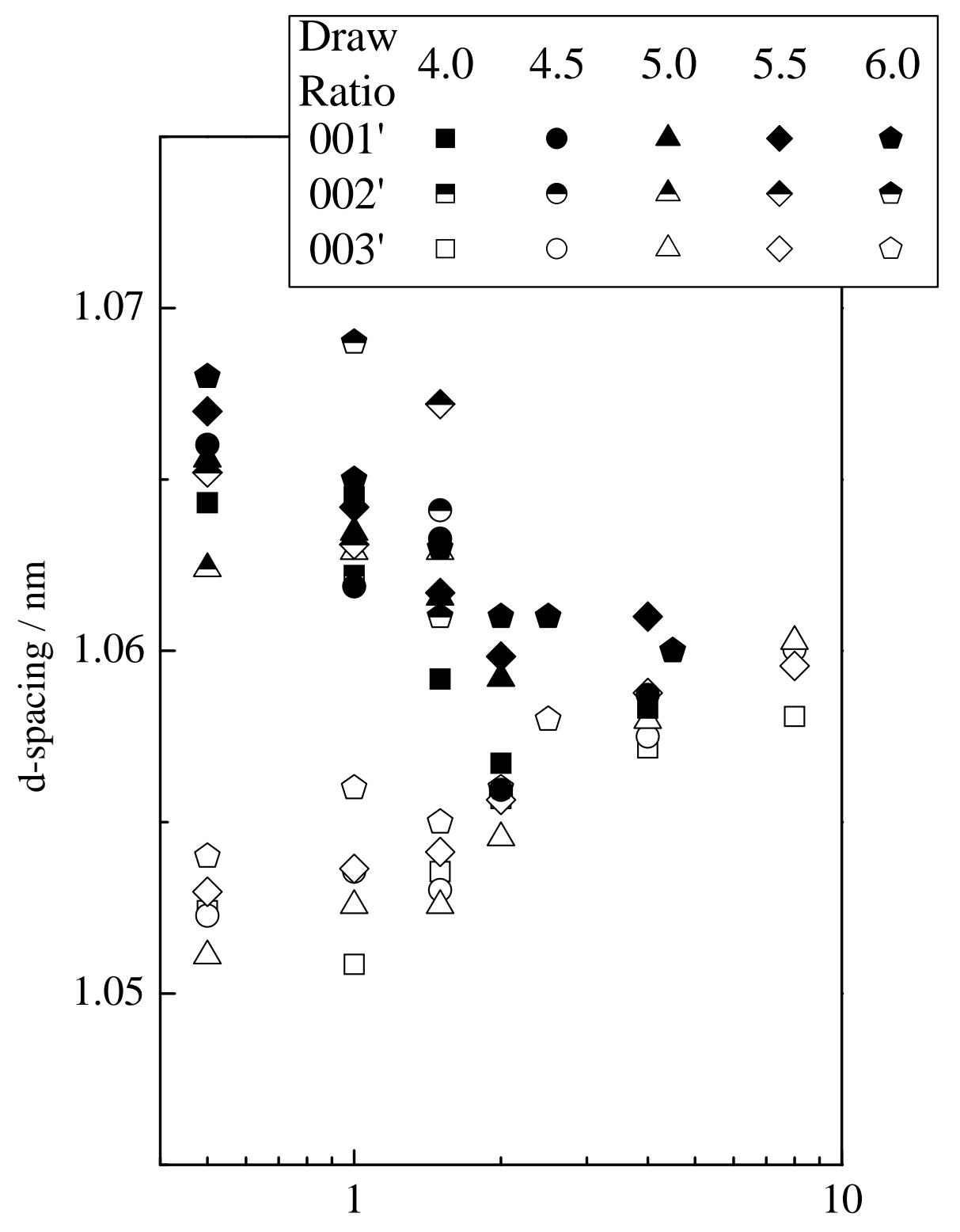

Elapsed Time after Necking / ms

Fig. 8 


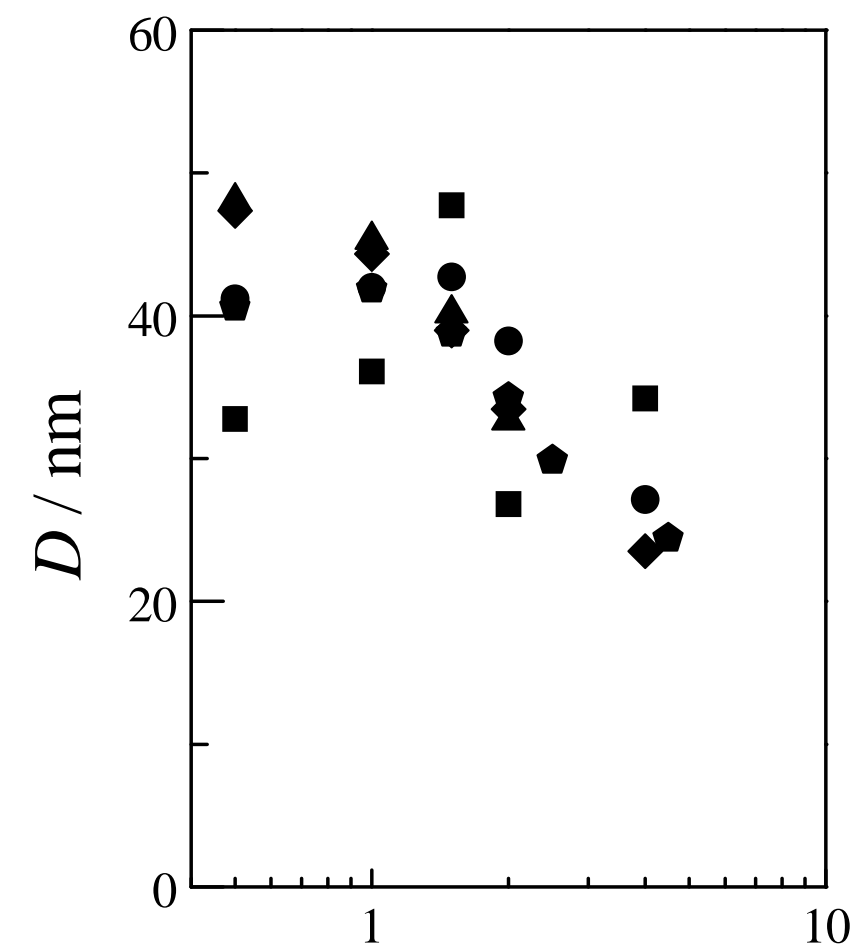

Elapsed Time after Necking / ms

Fig. 9 


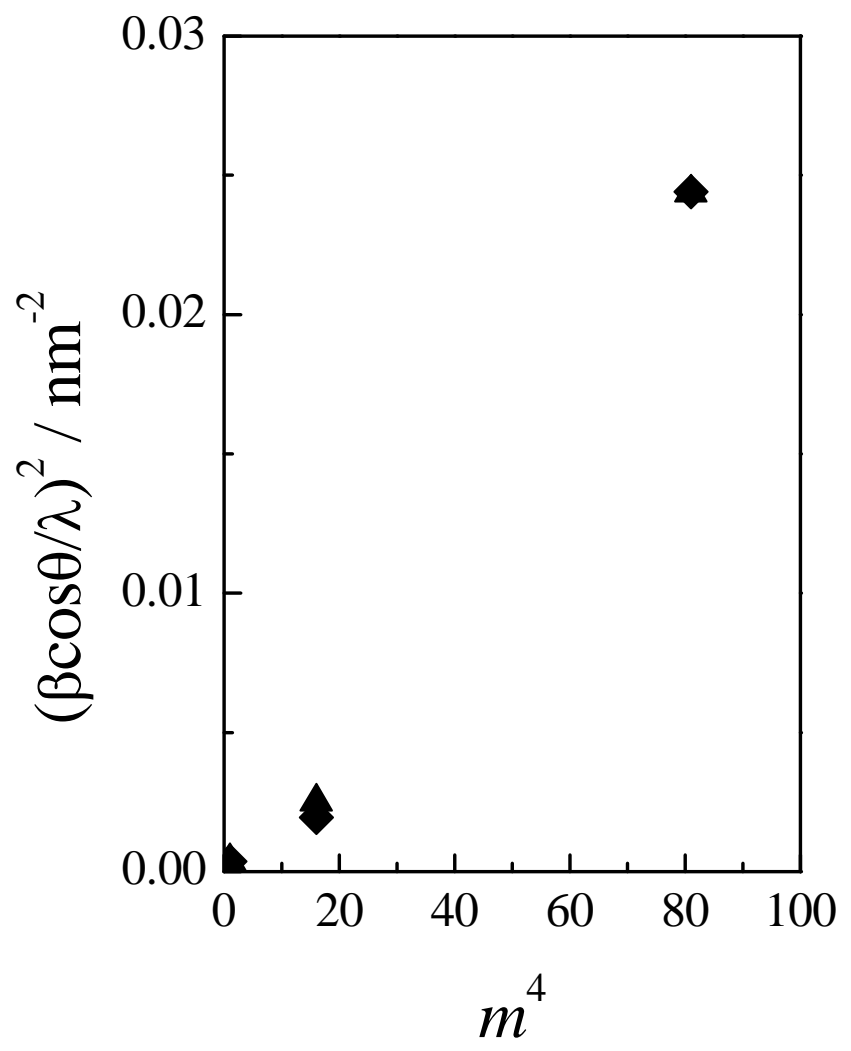

Fig. 10 


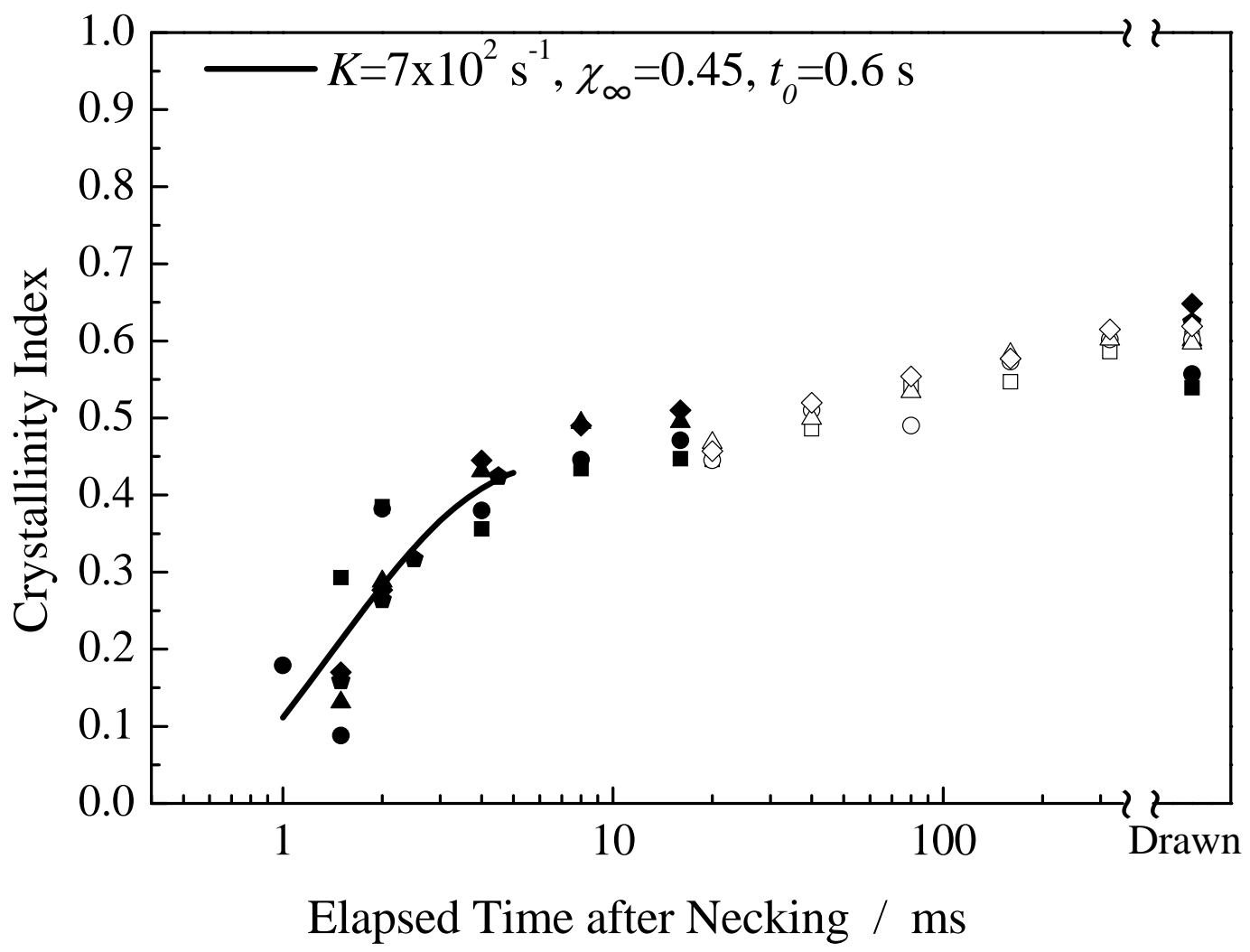

Fig. 11 


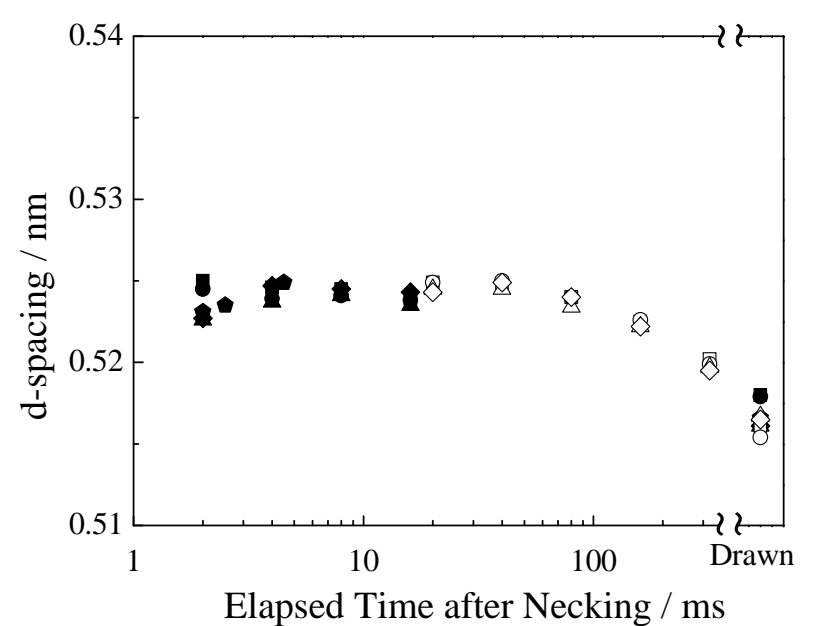

(a) (010)

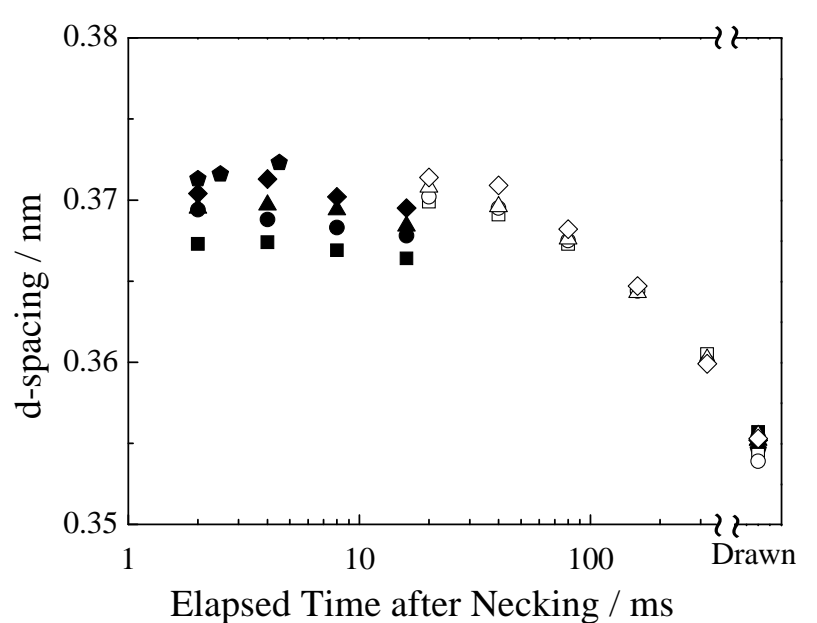

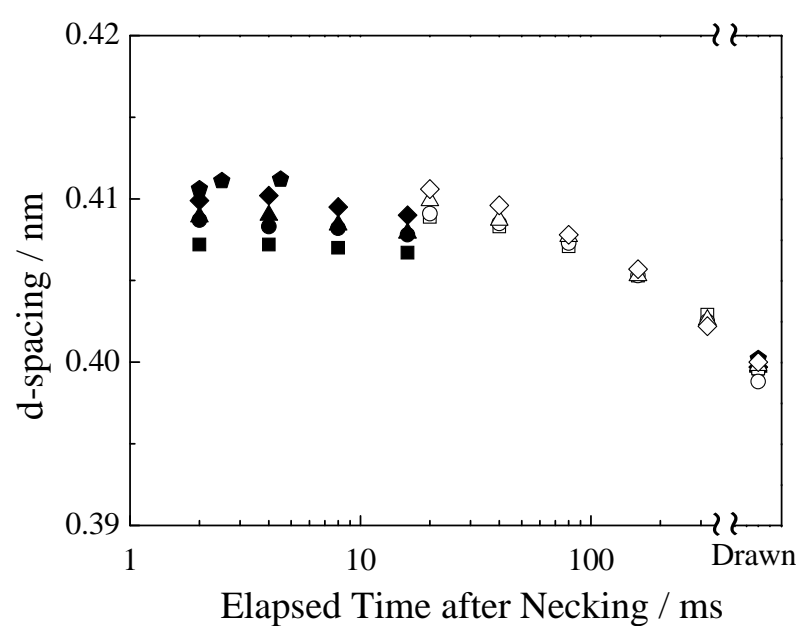

(b) (110)

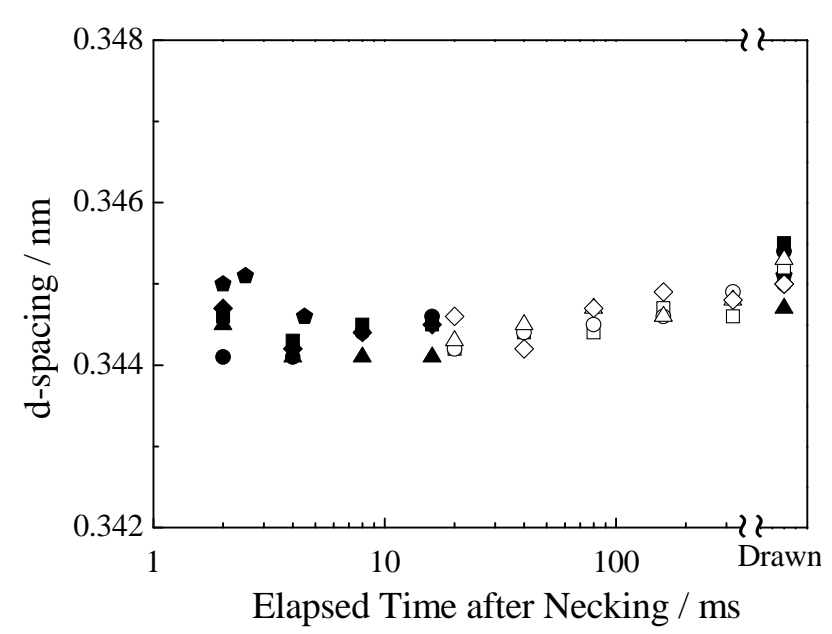

(d) (103)

Fig. 12 


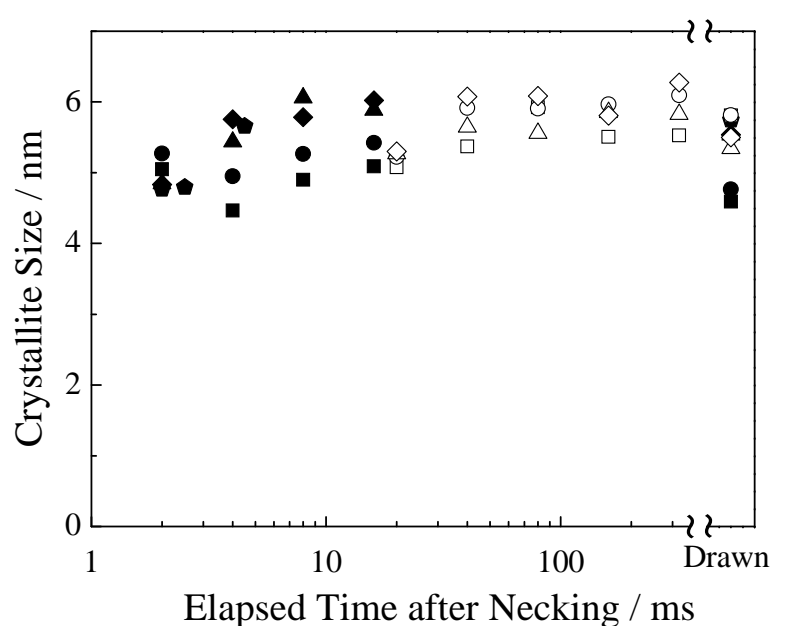

(a) (010)

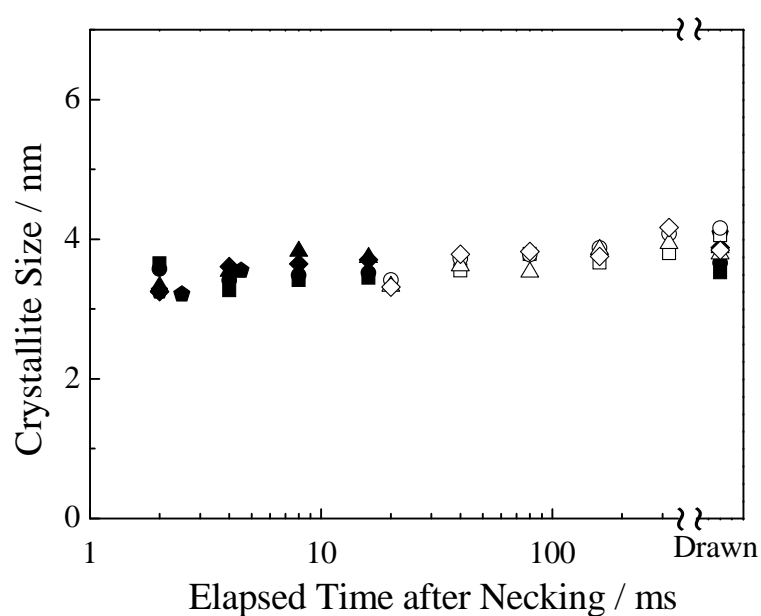

(c) $(100)$

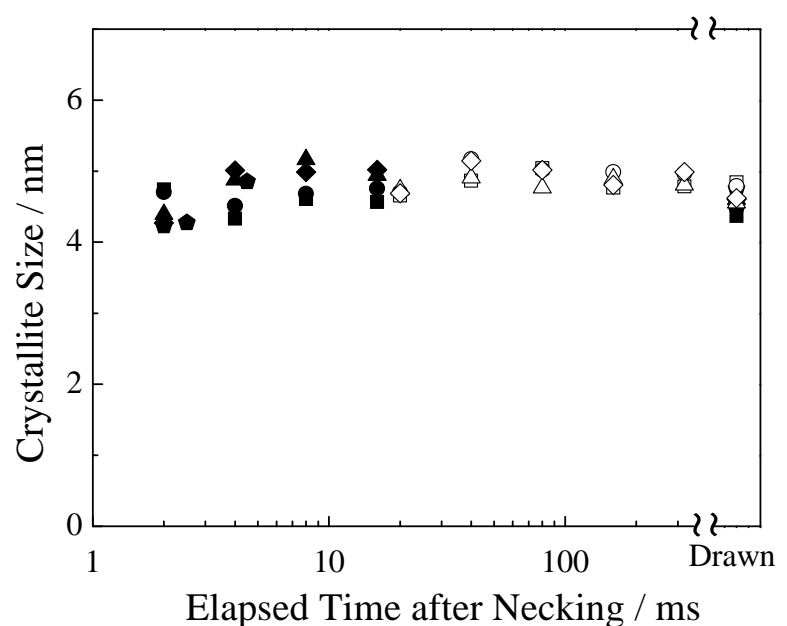

(b) $(110)$

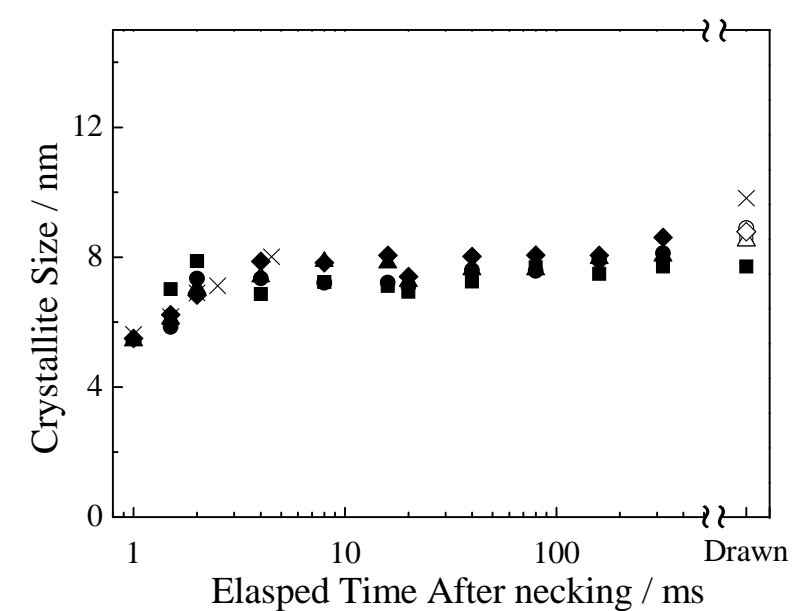

(d) $(103)$

Fig. 13 


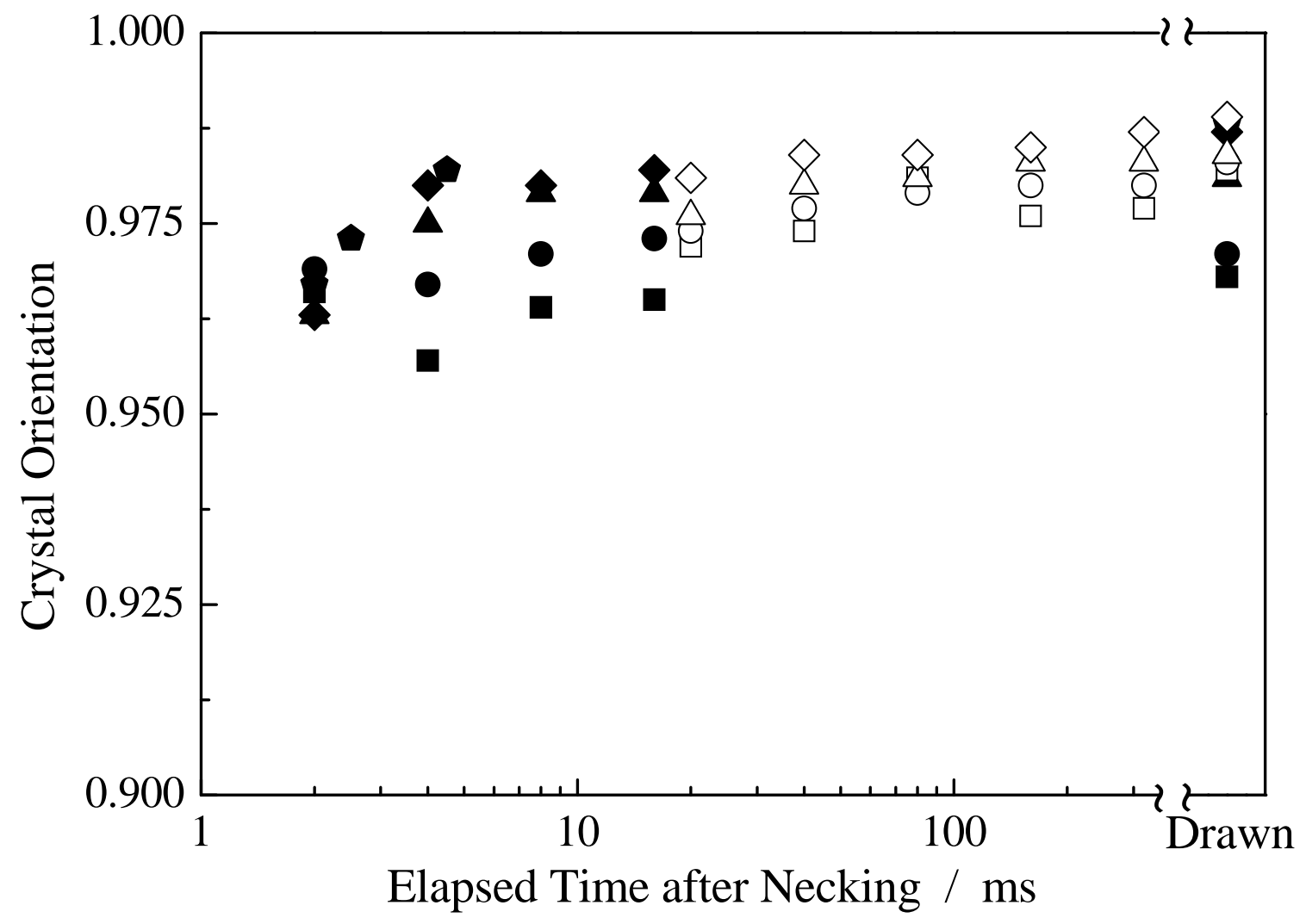

Fig. 14 


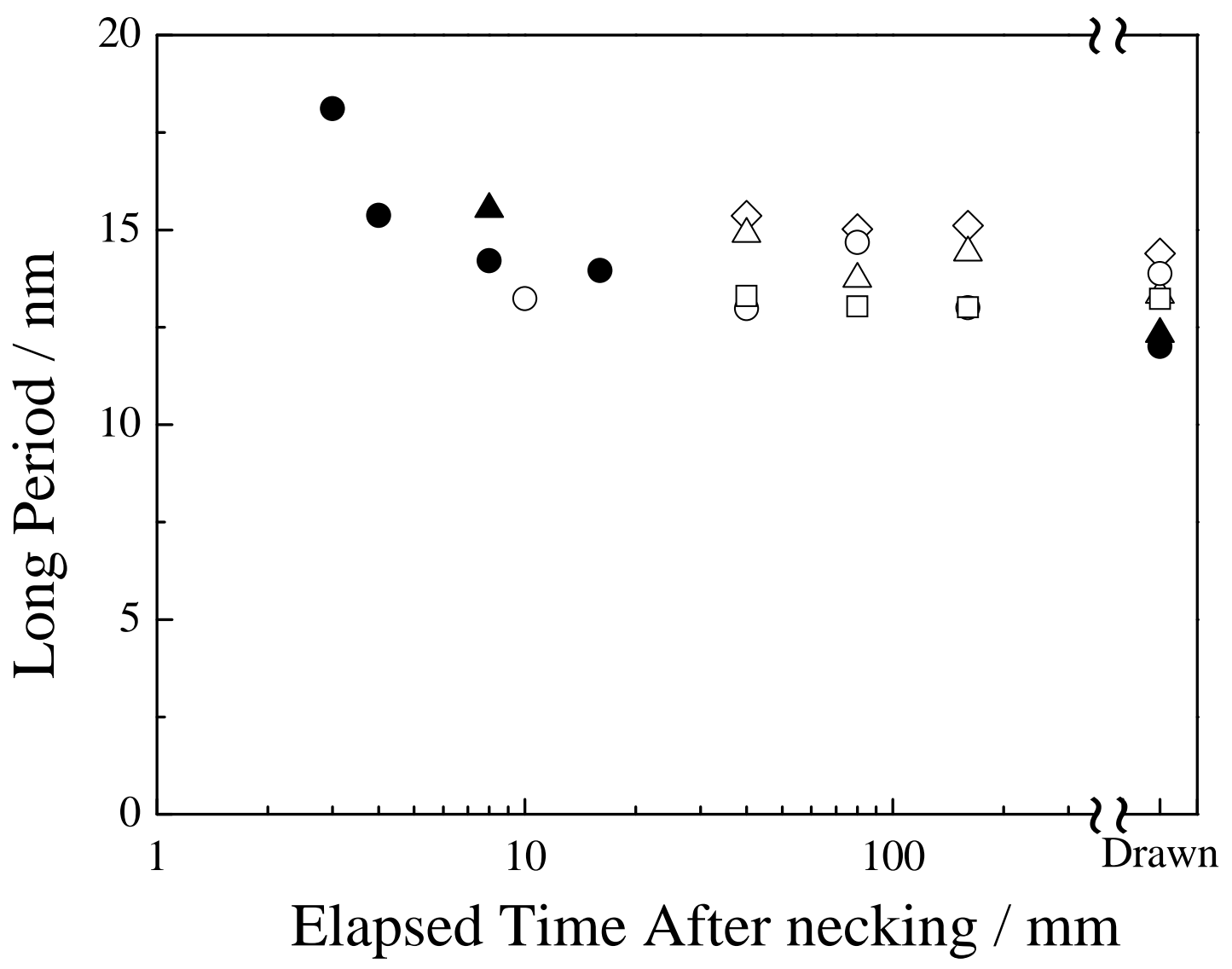

Fig. 15 


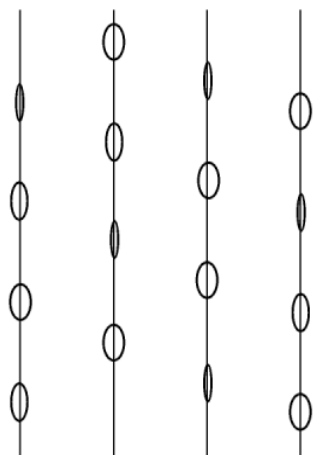

(a) One-Dimensional Order

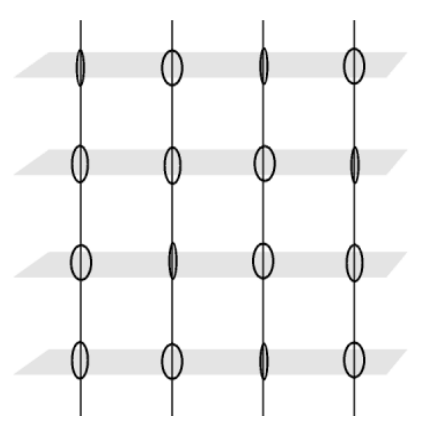

(b) Two-Dimensional Order

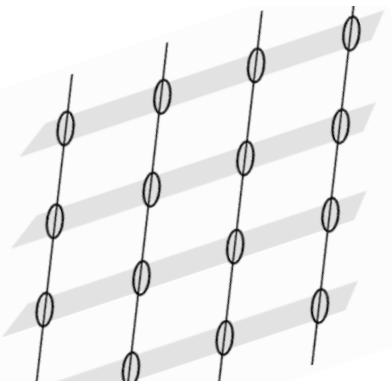

(c) Three-Dimensional Order

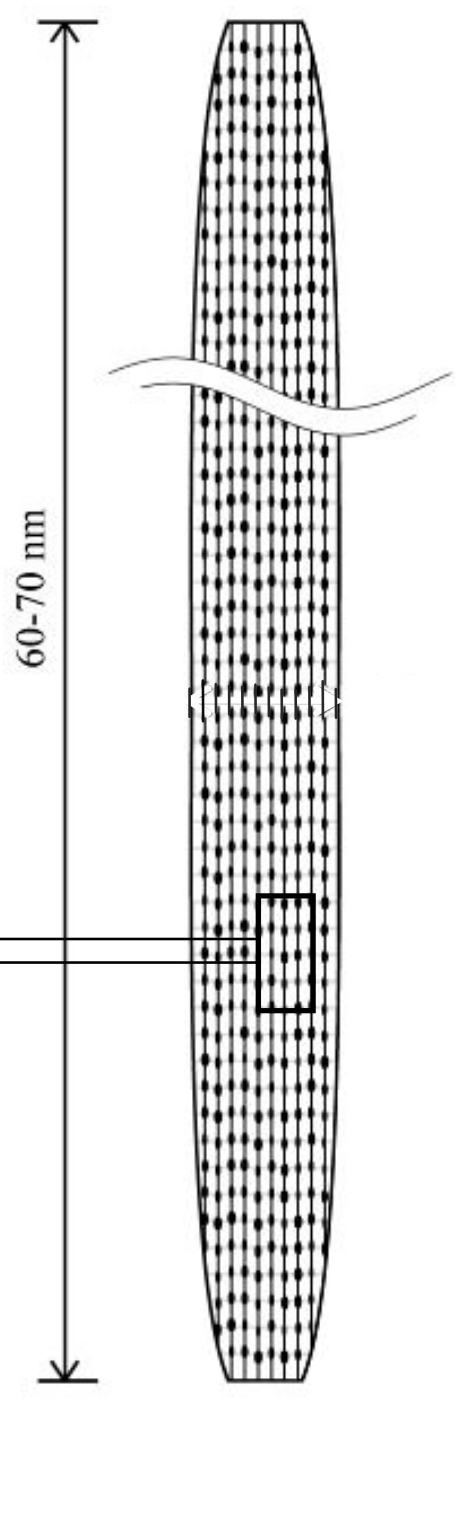

(d) Fibrillar Structure

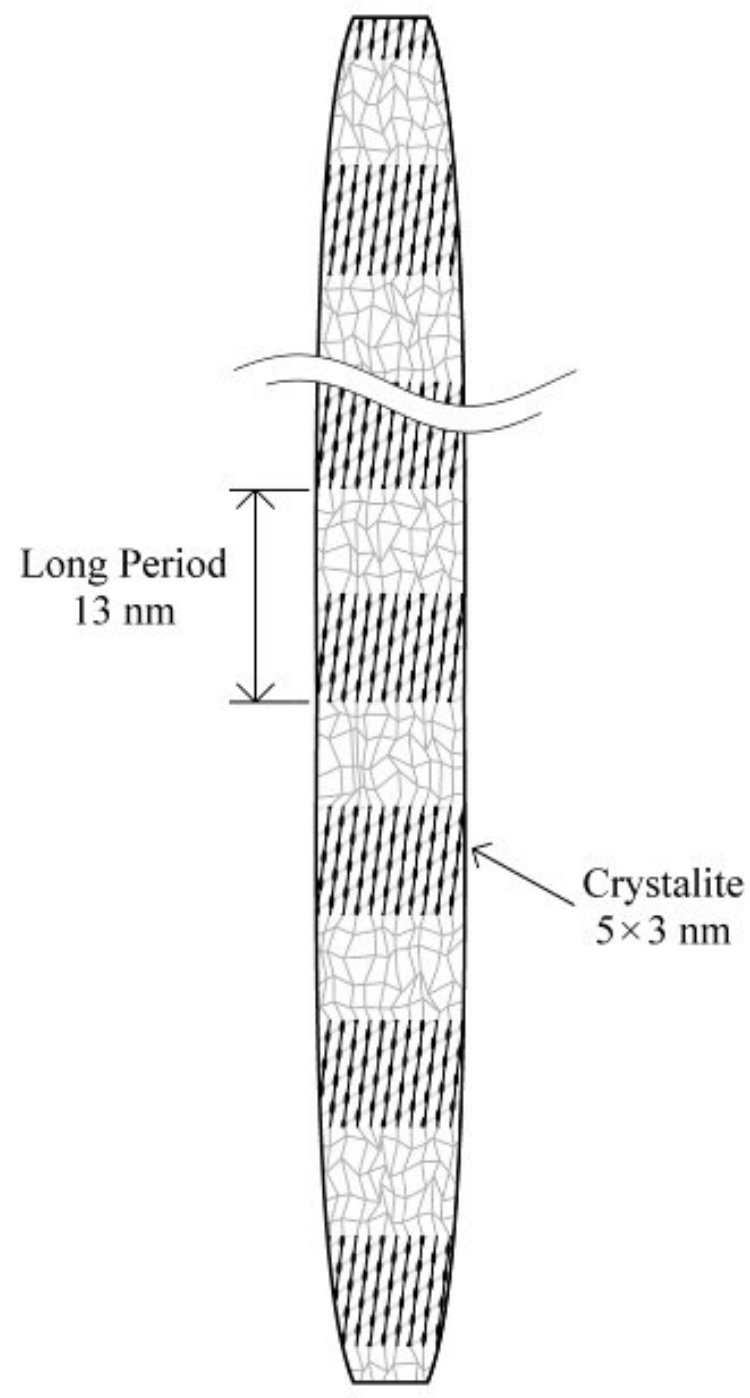

(e) Microfibril

Fig. 16 


\section{Figure Captions}

Figure 1 Schematic diagram of in-situ measurement system. The elapsed time after necking can be estimated from the fiber running speed $v$ and the distance $x$ between the necking point and measuring point.

Figure 2 Temperature profiles along the fiber for a take-up speed of $48 \mathrm{~m} / \mathrm{min}$ (a), and $6 \mathrm{~m} / \mathrm{min}$ (b). Profiles along the elapsed time for each take-up speed are also shown in (c) and (d). The draw ratio is $4.0(\square), 4.5(\bigcirc), 5.0(\triangle)$, and $5.5(\diamond)$. Figures (a) and (c) also show the estimated temperature profiles.

Figure 3 Transmitted x-ray intensity profiles for a take-up speed of $48 \mathrm{~m} / \mathrm{min}$ (a) and $6 \mathrm{~m} / \mathrm{min}$ (b). The draw ratio is $4.0(\square), 4.5(\bigcirc), 5.0(\triangle)$, and $5.5(\diamond)$. Necking positions determined by the temperature profiles shown in Figure 2 are plotted on the corresponding intensity with filled symbols.

Figure 4 WAXD patterns for varying draw ratio and elapsed time after necking. The fiber axis is oriented vertically in these images.

Figure 5 Intensity profiles along the equatorial (a), (c) and meridional (b) directions for a take-up speed of $48 \mathrm{~m} / \mathrm{min}$ (a), (b) and $6 \mathrm{~m} / \mathrm{min}$ (c). The elapsed time after necking is indicated in the figures.

Figure 6 SAXS patterns for varying draw ratio and elapsed time after necking.

Figure 7 WAXD patterns for a draw ratio of 5.5 and elapsed time of $0.5 \mathrm{~ms}$ after necking.

Figure 8 Interplanar spacing for meridional diffractions. For the (002') and (003') planes, two and three times the spacing are plotted. 
Figure 9 Length of the fibrillar structure estimated for (001') diffraction by Eq. 4 . The draw ratio is 4.0 (ם), $4.5(\bullet), 5.0(\mathbf{\Delta}), 5.5(\bullet)$ and $6.0(\bullet)$.

Figure 10 Hosemann plot (Eq. 3) for meridional diffractions, i.e. (001'), (002') and (003'). The draw ratio is $5.0(\boldsymbol{\Delta})$ and $5.5(\bullet)$, and the elapsed time is $0.5 \mathrm{~ms}$.

Figure 11 Crystallinity index obtained by equatorial intensity profiles. The draw ratio is $4.0(\mathbf{\square}), 4.5(\mathbf{\bullet})$, $5.0(\boldsymbol{\Delta}), 5.5(\bullet)$ and $6.0(\bullet)$. The closed symbols indicate the results for $48 \mathrm{~m} / \mathrm{min}$, while the open symbols indicate those for $6 \mathrm{~m} / \mathrm{min}$. The crystallinity calculated by Eq. 8 using the data indicated is also shown in the figure.

Figure 12 Interplanar spacings calculated for the (010), ( $\overline{1} 10),(100)$ and ( $\overline{1} 03)$ diffractions. The symbols are the same as those in Figure 11.

Figure 13 Crystallite sizes calculated for the (010), ( 110$),(100)$ and ( $\overline{1} 03)$ diffractions. The symbols are the same as those in Figure 11.

Figure 14 Crystal orientation factor calculated by the (010), ( $\overline{1} 10)$, and (100) diffractions. The symbols are the same as those in Figure 11.

Figure 15 Long Period obtained by SAXS image. The symbols are the same as those in Figure 11.

Figure 16 Model of fiber structure development. The oriented amorphous phase (a) formed by necking deformation transformed into a two-dimensionally ordered structure (b) within less than $0.5 \mathrm{~ms}$, and subsequently transformed into a three-dimensionally ordered triclinic crystal (c) 1 to $4 \mathrm{~ms}$ after the deformation. 